\title{
Raman Spectroscopy on Semiconductor Nanowires
}

\author{
Ilaria Zardo ${ }^{1}$, Gerhard Abstreiter ${ }^{1}$ and Anna Fontcuberta i Morral ${ }^{1,2}$ \\ ${ }^{1}$ Walter Schottky Institut and Physik Department, Technische Universität München \\ 2Laboratoire des Matériaux Semiconducteurs, Ecole Polytechnique Fédérale de Lausanne \\ ${ }^{1}$ Germany \\ 2Switzerland
}

\section{Introduction}

Raman scattering is an inelastic light scattering non-destructive technique which allows the access mainly to the phonon modes at the $\Gamma$ point of materials and in some cases to the dispersion (Goni A.R. et al., 2001; Zunke et al., 1995; Weinstein et al., 1975). Since its discovery, Raman has been used both for the characterization of materials and for the understanding of basic interactions such as plasmonic excitations (Raman et al., 1928; Szymanski H.A. et al., 1967; Otto et al., 1992; Schuller et al., 1996; Steinbach et al., 1996; Ulrichs et al., 1997, Sood et al 1985, Abstreiter et al. 1979, Roca et al. 1994, Pinczuk et al 1977, Pinczuk et al., 1979). Raman spectroscopy can be experimentally performed at the nanoscale by using a confocal microscope or even a tip enhanced scanning microscope. It is possible to obtain lateral submicron resolutions of the properties of a material (Hartschuh et al., 2003). Nowadays Raman spectroscopy is a versatile and relative standard tool for the characterization of materials giving detailed information on crystal structure, phonon dispersion, electronic states, composition, strain and so-on bulk materials, thin film and nanostructures (Cardona, 1982; Anastassakis, 1997; Reithmaier et al., 1990; Spitzer et al., 1994; Pinczuk et al., 1977; Pinczuk et al., 1979; Baumgartner et al., 1984; Schuller et al., 1996; Pauzauskie et al., 2005; Long, 1979).

In the last decade Raman spectroscopy has been increasingly used to study nanowires and quantum dots (Abstreiter et al., 1996; Roca et al., 1994). Several new phenomena have been reported to date with respect to one-dimensional structures. For example, the high surfaceto-volume ratio has enabled the measurement of surface phonon modes (Gupta et al., 2003a; Krahne et al 2006; Adu et al., 2006; Spirkoska et al., 2008). Some authors report a increase in the scattered intensity for nanoscale structures with respect to their bulk counterpart, effect denominated as 'Raman antenna effect' (Xiong et al., 2004; Xiong et al., 2006; Cao et al., 2007). Additionally, polarization dependent experiments on single carbon nanotubes and/or nanowires have shown that the physics behind Raman scattering of such one-dimensional nanostructures can differ significantly from the bulk (Frechette et al., 2006; Livneh et al., 2006; Cao et al., 2006). Indeed, the highly anisotropic shape of the nanowires can lead to angular dependencies of the modes which otherwise would not be expected from selection rules (Frechette et al., 2006; Livneh et al., 2006; Cao et al 2006).

Source: Nanowires, Book edited by: Paola Prete,

ISBN 978-953-7619-79-4, pp. 414, March 2010, INTECH, Croatia, downloaded from SCIYO.COM 
Overall, Raman spectroscopy of nanostructures represents an extremely active and exciting field for the benefit of science and technology at the nanoscale. The arising new phenomena and technical possibilities open new avenues for the characterization of materials but also for the understanding of fundamental process in nanoscale matter. In this chapter, we provide a review of Raman spectroscopy on nanowires, in which an overview of the selection rules, appearance of new modes and size effects will be given.

\section{Selection rules in Raman scattering of nanowires}

\subsection{Raman selection rules, application to the geometry of nanowires}

Raman scattering is a manifestation of the interaction between the electromagnetic radiation and vibrational and/or rotational motions in a material. It provides information about the symmetry and composition of the system, the lattice dynamics, structural transitions, strain and electronic states. The scattering process involves two energy quanta simultaneously. It is usually schematized in two steps:

i. A photon with energy $h v_{o}$ and wave vector $\vec{q}$ is absorbed, exciting the system from a initial state 1 to a state $n$;

ii. The system emits a photon with energy $h v^{\prime}$ and wave vector $\vec{q}^{\prime}$ and relaxes from the state $n$ to a final state 2 .

In the case where the final state is identical to the initial one the incident and scattered light have the same frequency $\left(v^{\prime}=v_{o}\right)$. This process is called elastic or Rayleigh scattering. When the final state is different from the initial one, the scattering process is inelastic. In this case, the creation or the annihilation of an excited state of the system occurs, and the emitted photon has lost or gained energy. These processes are called respectively Stokes scattering and anti-Stokes Raman scattering. For the conservation of energy, the frequency of the scattered radiation is given by:

$$
v^{\prime}=v_{o} \pm \frac{E_{1}-E_{2}}{h}=v_{o} \pm v_{v}
$$

with $v_{o}$ the frequency of the incoming photon, $v^{\prime}$ the frequency of the emitted photon, $E_{1}$ and $E_{2}$ the energies of the initial and final state of the system. These scattering processes are schematically illustrated in Fig. 1.

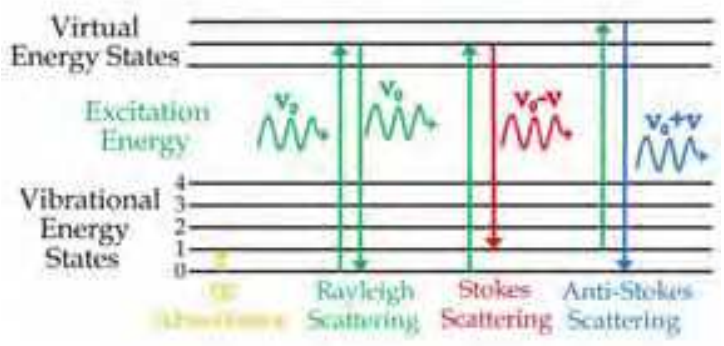

Fig. 1. Schematic drawing of transitions between generic vibrational energy states due to, from left to right, infrared absorbance, Rayleigh Scattering Stokes Raman Scattering and anti-Stokes Raman Scattering. 
The difference between the Raman scattering frequency $v^{\prime}$ and the excitation frequency $v_{o}$ is independent from the last one and it's equal to $\pm v^{n}$. The probability of the Stokes scattering and the anti-Stokes scattering is different, because of the different population of the two energy levels at a temperature T. The intensity of the Raman lines is proportional to the transition probability. For this reason the Stokes lines are more intense than the antiStokes. In the following, a short description of the Raman effect is given within the classical picture (Turrel \& Corset, 1996). In this frame, spatial and temporal fluctuations of the electronic contributions of the polarizability are at the origin of the Raman scattering. The electric field originated by a plane monochromatic wave with wave vector $\vec{K}_{o}$ in a point $\vec{r}$ in space in a transparent crystal is given by:

$$
\vec{E}=E_{o}\left\{\exp -2 \pi i\left(\vec{K}_{o} \cdot \vec{r}-v_{o} t\right)\right\}
$$

with $v_{o}$ the frequency of the light and $t$ the time. The electric field causes an induced dipole moment given by:

$$
\vec{\mu}=\alpha \vec{E}
$$

where $\alpha$ is the polarizability. Both $\vec{\mu}$ and $\vec{E}$ are vectors, while $\alpha$ is a $3 \times 3$ tensor with real elements, unless magnetic phenomena are involved. The coordinate system and the symmetry of the crystal determine the tensor form. Due to the time dependency of $\vec{\mu}$ and $\vec{E}$, the induced dipole moment will oscillates in time, with consequent radiation emission. The polarizability can be expanded in as a Taylor series in the normal coordinates $Q_{K}=Q_{K}^{0} \exp ^{-2 \pi i\left[\vec{K}_{K} \cdot \vec{r}-v_{K} t\right]}$, with $\vec{K}_{K}$ the wave vector of lattice wave $K$. Equation (3) becomes:

$$
\vec{\mu}=\alpha_{0} E_{o} \exp ^{-2 \pi i\left(\vec{K}_{o} \cdot \vec{r}-v_{o} t\right)}+E_{o} \sum_{K} \alpha_{K}^{\prime} Q_{K} \exp ^{-2 \pi i\left[\left(\vec{K}_{0} \pm \vec{K}_{K}\right) \cdot \vec{r}-\left(v_{0} \pm v_{K}\right) t\right]}
$$

with $\alpha_{K}^{\prime}=\left(\frac{\partial \alpha}{\partial \boldsymbol{Q}_{K}}\right)_{0}$.

The first term of equation (4) describes the Rayleigh scattering, the oscillation of the induced dipole at the same frequency of the incident light. The second term represents the dipole oscillating at a frequency shifted by the frequency of the normal modes. Therefore, $v_{o} \pm v_{K}$ is the frequency of the scattered light, which propagates in the direction $\vec{K}_{0} \pm \vec{K}_{K}$.

The Raman scattering is governed by the conservation of energy and by conservation of momentum, which implies that $\vec{K}_{0}=\vec{K}_{S} \pm \vec{K}_{K}$, being $\vec{K}_{S}$ the wave vector of the scattered light. Namely, the orientation of the crystallographic axes with respect to the direction and polarization of the scattered light affects the Raman spectrum. In this respect, it is evident that Raman spectroscopy on single crystals gives information about the crystal symmetry. The intensity of the scattered light $I_{s}$, which is the scattered energy per unit time, into a solid angle $d \Omega$ is given by:

$$
I_{S}=I_{i} \cdot k \cdot\left|\hat{e}_{i} \cdot \ddot{R} \cdot \hat{e}_{s}\right|^{2} d \Omega
$$

with $I_{i}$ the irradiance - energy per unit area per unit time - of the excitation incident on the sample, $k=4 \pi^{2} a^{2} v_{s}^{-4}, a \approx 1 / 137, v_{s}$ the wavenumber of the scattered light, $\hat{e}_{i}\left(\hat{e}_{s}\right)$ the 
polarization unitary vectors of the incident (scattered) light and $\vec{R}$ the scattering tensors. It is worth to note that the symmetry properties of the polarizability and the scattering tensors are the same. In the case where Raman scattering is realized at the submicron scale, a microscope objective has to be used. For simplicity, a backscattering configuration is preferred. In this configuration the scattered light is collected along the same direction of the excitation, as shown schematically in Fig. 2.

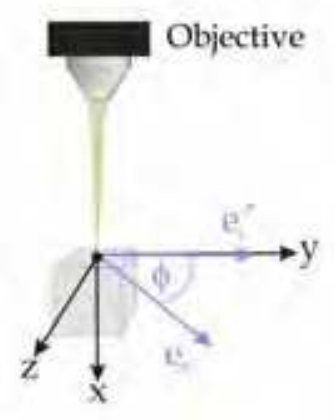

Fig. 2. Schematic drawing of the backscattering geometry. The incoming light is directed along the $\mathrm{x}$ direction, with the polarization directed along the $\mathrm{y}$ direction. The scattered light is collected along the $\mathrm{x}$ direction and its polarization has components along the $\mathrm{y}$ and $\mathrm{z}$ directions.

As an example, we consider the backscattering geometry with the set of axes as depicted in Fig. 2 and $\hat{e}_{i}|| y$, the Raman intensity is calculated as follows:

$$
\left.I_{s} \propto(010)\left(\begin{array}{lll}
R_{x x} & R_{x y} & R_{x z} \\
R_{y x} & R_{y y} & R_{y z} \\
R_{z x} & R_{z y} & R_{z z}
\end{array}\right)\left(\begin{array}{l}
0 \\
1 \\
1
\end{array}\right)\right|^{2}=\left|R_{y y}+R_{y z}\right|^{2}
$$

The use of a determined incident and analyzed polarization in the Raman scattering experiments results in the selection of certain elements of $\vec{R}$. In this way, polarized Raman spectroscopy enables the determination of the Raman selection rules and the tensor symmetry. In the measurements on a single bulk crystal, it is much more convenient to use the crystallographic axis as a basis and express the polarizability tensor in this basis.

As an example, we consider zinc-blende GaAs. The phonon dispersion is composed of 6 different branches: two transverse and one longitudinal acoustical modes (TA and LA) as well as two transverse and one longitudinal optical modes (TO and LO). The optical photon modes are usually indicated $\mathrm{E}_{1}(\mathrm{TO})$ and $\mathrm{A}_{1}(\mathrm{LO})$. The notation $\mathrm{E}_{1}$ and $\mathrm{A}_{1}$ denote respectively to modes vibrating perpendicular and along the $\mathrm{z}$ axis. The Raman tensors for zinc blende GaAs are usually given in the base $x=(100), y=(010)$ and $z=(001)$, resulting in:

$$
R(x)=\left(\begin{array}{lll}
0 & 0 & 0 \\
0 & 0 & 1 \\
0 & 1 & 0
\end{array}\right), R(y)=\left(\begin{array}{ccc}
0 & 0 & 1 \\
0 & 0 & 0 \\
1 & 0 & 0
\end{array}\right) \text { and } R(z)=\left(\begin{array}{lll}
0 & 1 & 0 \\
1 & 0 & 0 \\
0 & 0 & 0
\end{array}\right)
$$


In this set of axes and in backscattering geometry with the direction of excitation and collection perpendicular to a $\{001\}$ plane, the TO is forbidden while the LO mode is allowed. The intensity of the scattered light polarized along the $x$ or $y$ direction as a function of the angle between the polarization of the excitation and the $x=(100)$ can be calculated using equation (6). The theoretical azymutal dependence of the intensities is plotted in Fig. 3.

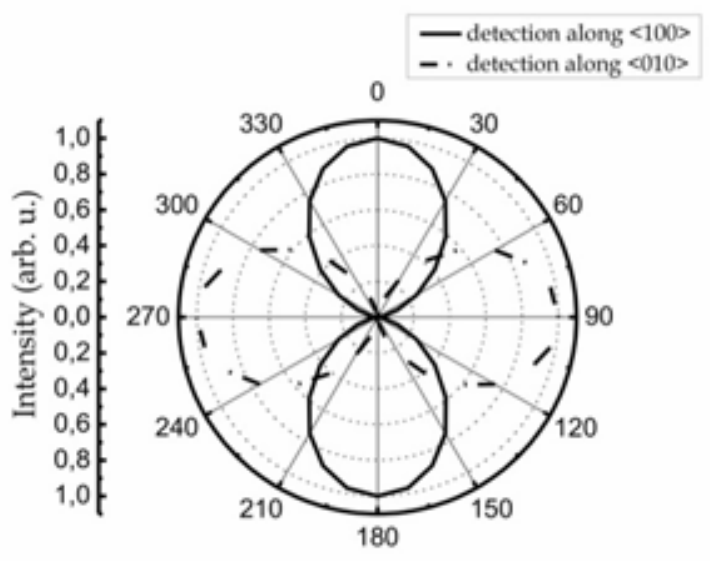

Fig. 3. Theoretical azimuthal dependence of the LO mode of a bulk GaAs (001). Continuous and dashed lines represent the components along the [100] and [010] of the Raman signal, respectively.

The selection rules and the dependency of the intensity on the polarization direction can be calculated even from the other surfaces in the same way. In backscattering geometry both the $A_{1}(L O)$ or $E_{1}(T O)$ are observed from the (111) surfaces, while only $E_{1}(T O)$ is observed from the (110) planes.

If we now consider the geometry of the nanowires, the important crystallographic axis correspond to the directions $x=(0-11), y=(211)$ and $z=(-111)$, which should be used as a basis. A schematic drawing of the relevant axes on a reference bulk sample is shown in Fig. 4a: the $x$ axis corresponds the direction of the incident and scattered light in the [0-11] direction, while $y$ and $z$ are the in plane axes respectively parallel to [211] and [-111]. The selection rules are obtained by transforming the Raman tensor and by expressing the polarization vectors into the new basis and, using eq. (6). The values of the Raman tensor for the transversal modes in that configuration for incident light along the $\mathrm{x}$ axis are:

$$
R^{\prime}(y)=\left(\begin{array}{ccc}
0 & 1 / \sqrt{3} & -1 / \sqrt{6} \\
1 / \sqrt{3} & 2 / 3 & -1 / 3 \sqrt{2} \\
-1 / \sqrt{6} & 1 / 3 \sqrt{2} & -2 / 3
\end{array}\right), R^{\prime}(z)=\left(\begin{array}{ccc}
0 & -1 / \sqrt{3} & 1 / \sqrt{6} \\
-1 / \sqrt{3} & 2 / 3 & 1 / 3 \sqrt{2} \\
1 / \sqrt{6} & 1 / 3 \sqrt{2} & -2 / 3
\end{array}\right)
$$

The intensity of the scattered light polarized parallel or perpendicular to the [-111] direction, $I_{s}(\|)$ and $I_{s}(\perp)$, as a function of the angle $\alpha$ between the polarization of the excitation with the [-111] axis is: 


$$
\begin{gathered}
I_{s}(\perp)=\left|\left(\begin{array}{lll}
0 & \sin \alpha & \cos \alpha
\end{array}\right)\left[R^{\prime}(y)\left(\begin{array}{l}
0 \\
1 \\
0
\end{array}\right)+R^{\prime}(z)\left(\begin{array}{l}
0 \\
1 \\
0
\end{array}\right)\right]\right|^{2}=\left|\frac{4}{3} \sin \alpha+\frac{2 \cos \alpha}{3 \sqrt{2}}\right|^{2} \\
I_{s}(\|)=\left|\left(\begin{array}{lll}
0 & \sin \alpha & \cos \alpha
\end{array}\right)\left[R^{\prime}(y)\left(\begin{array}{l}
0 \\
0 \\
1
\end{array}\right)+R^{\prime}(z)\left(\begin{array}{l}
0 \\
0 \\
1
\end{array}\right)\right]\right|^{2}=\left|\frac{2}{3 \sqrt{2}} \sin \alpha+\frac{4}{3} \cos \alpha\right|^{2}
\end{gathered}
$$

Fig. $4 \mathrm{~b}$ and $\mathrm{c}$ show the theoretical and experimental dependency of the two components of the scattered light, respectively.
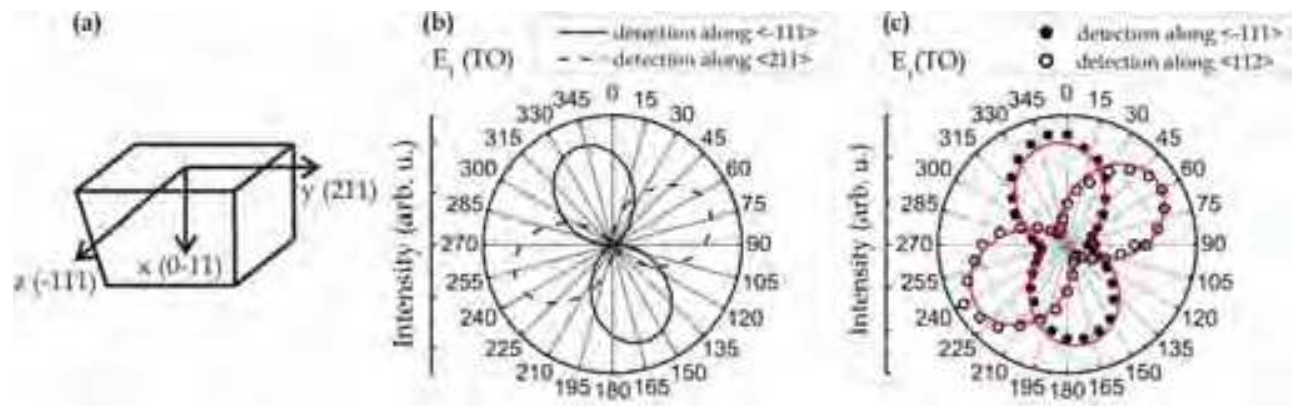

Fig. 4. (a) Crystal facets of the reference used for the measurement of the selection rules in GaAs. The axis correspond to the crystallographic directions: $x=(0-11), y=(211)$ and $z=(-111)$. (b) Theoretical azimuthal dependence of the TO mode of a bulk GaAs (0-11), as in (a). Continuous and dashed lines represent the components along the [-111] and [211] of the Raman signal, respectively. (c) Measured azimuthal dependence of the TO mode of a bulk GaAs (0-11). Diamonds and open circles represent the components along the [-111] and [211] of the Raman signal, respectively. The continuous line is a squared sine fit to the data, which describes polar behaviour.

As mentioned above, this set of axis is the one that should be used for the investigation of single GaAs nanowires with [111] growth axis. For further clarity, a schematic drawing of the nanowire with the corresponding set of axis, as used in Raman backscattering experiment, is presented in Fig. 5a. We studied nanowires presenting a mixture of zinc blende and wurtzite structure. In this case, a further optical mode can be observed at $\mathrm{k}=0$, namely the $\mathrm{E}_{2} \mathrm{H}$ (see section 4). Fig. 5b shows representative Raman spectra realized under the main four polarization configurations. The azymuthal dependence of $\mathrm{E}_{1}(\mathrm{TO})$ and $\mathrm{E}_{2} \mathrm{H}$ is presented in Fig. $5 \mathrm{c}$ and $\mathrm{d}$. The scattered light has been analyzed selecting the components with polarization parallel $-I_{s}(\|)$ - and perpendicular $-I_{s}(\perp)$ - to the $z$ axis. The $E_{1}(T O)$ mode is polarized along the axis of the nanowire. Interestingly, also $I_{s}(\perp)$ seems to have a slighty higher intensity when the incident light is polarized along the nanowire axis. The scattered light with polarization perpendicular to the $\mathrm{z}$ axis, exhibits a drop in the intensity, compared to the measurements on GaAs bulk (Fig. 4c). Indeed, the ratio of intensity between $I_{s}(\|)$ and $\mathrm{I}_{\mathrm{s}}(\perp)$ is about 5 . Interestingly, the azymuthal dependence of the $\mathrm{E}_{2}{ }^{\mathrm{H}}$ mode associated with the 
wurtzite phase exhibits a quite different behavior. In this case the maximum intensity of the scattered light is observed when the incident light is perpendicular to the nanowire axis, both for Is $(\|)$ and Is $(\perp)$-though for the latter the dependence is less clear due to the low intensity-.
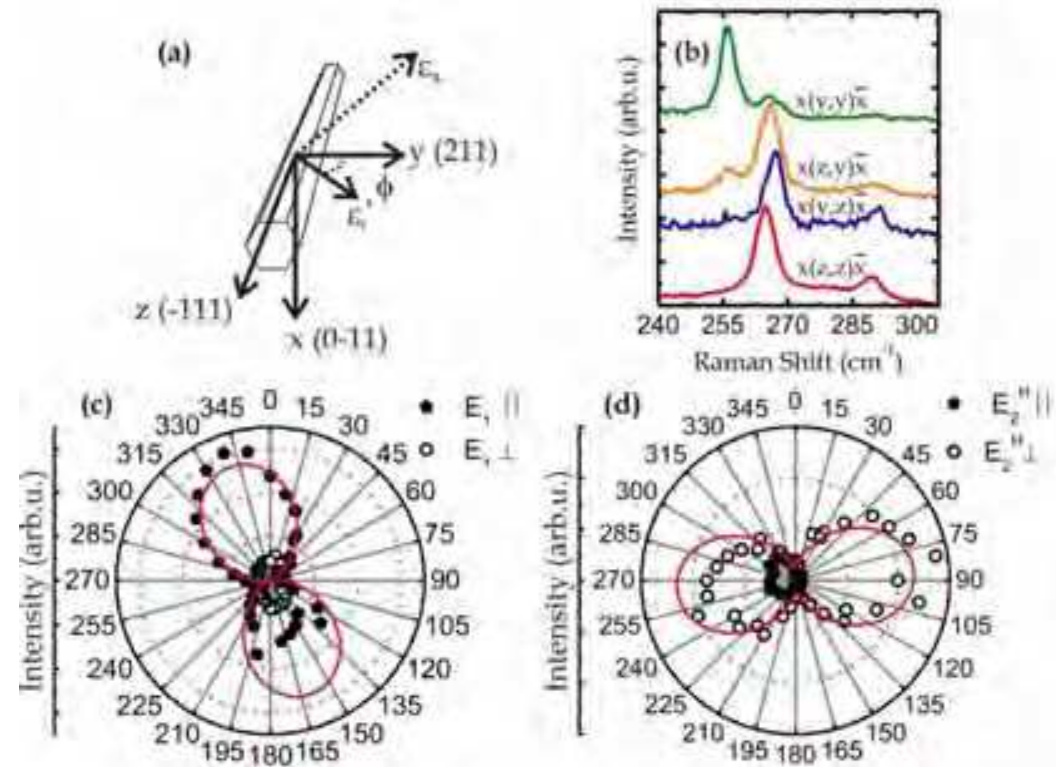

Fig. 5. (a) Sketch of the configurations used for the measurement of GaAs nanowires in backscattering geometry. The crystal facets of the nanowire and the corresponding set of axis used as indicated: $x=(0-11), y=(211)$ and $z=(-111)$. (b) Representative Raman spectra realized under the main four configurations. For better illustration, the spectra have been normalized and shifted vertically. All spectra have been collected in the same position of the nanowire. Azimuthal dependence of the $\mathrm{E}_{1}(\mathrm{TO})$ mode $(\mathrm{c})$ and of the $\mathrm{E}_{2} \mathrm{H}$ mode $(\mathrm{d})$, related to the wurtzite structure. Diamonds and open circles represent the parallel and perpendicular components of the Raman signal collected, respectively. The continuous lines are squared sine fit.

\section{$\rightarrow$ Effect of the dielectric mismatch}

In the case of nanowires, it is worth noticing that there is an enhanced response of the Raman scattering for polarizations along the nanowire axis. As it will be shown in the following, this is partly due to the one-dimensionality and to the small diameter of the nanowires, as it has been reported in literature (Cao et al., 2007; Livneh et al., 2007; Papadimitriou \& Nassiopoulou, 1998; Pauzauskie et al., 2005; Duesberg et al., 2006; Fréchette \& Carraro, 2006; Cao et al., 2006; Xiong et al., 2006). Xiong et al. found that nanowires with a diameter $d<<\lambda / 4$, with $\lambda$ the wavelength of the excitation, show a dipolar behavior. Namely, the Raman scattering intensity is $\sim I_{0} \cos ^{2} \alpha$, with $I_{0}$ the incident laser intensity and $\alpha$ the angle between the electric field of the laser and the nanowire axis. For larger diameters, $d>>\lambda / 4$, the nanowires present a multipolar character. The authors address the origin of this effect to the scattering of the electromagnetic field from a dielectric cylinder of 
nanoscale dimensions. The calculations show that the electric field inside the nanowires with bigger diameter is increased when the electric field of the excitation is either parallel or perpendicular to the nanowire axis. Instead, for smaller diameters, the electric field inside the nanowire is strongly suppressed when the electric field of the excitation is perpendicular to the nanowire axis. Experiments on silicon nanocones showed that the enhancement in the Raman scattering, due to the enhanced internal field, decreases with increasing the nanowires diameter and increases with the wavelength of the excitation, features which suggest a resonant nature (Cao et al., 2006). This enhancement in the Raman scattering is in analogy with absorption, photoluminescence and photocurrent measurements (Cao L., 2009; Wang J. 2009; Thunich S., 2009).

\subsection{Appearance of new modes: surface and breathing modes.}

Studies comparing Raman scattering experiments of bulk and nanostructured materials have been reported in literature for several different kind of systems. It is usually observed that the transversal optical (TO) and the longitudinal optical (LO) modes have a position in energy close to that observed in bulk. When scaling down the size and the dimensionality of the structures, the position can change (see section 3.1). Additionally, new Raman modes can be found. Effects related to the shape of the system can become significant. The existence of boundary conditions at the nanoscale gives rise to electric and polarization forces. The surfaces represent a new mechanical boundary, since the surface atoms are "less bound" and "feel" a different local field from the bulk. This has consequences even in the propagation of an optical phonon, where the oscillating dipoles - created by the out of phase oscillation of ions and cations - interact by a dipole-dipole interaction. Mahan et al. developed and presented a model which describes the variation of the long range dipolar interactions due to the nanowires geometry, leading to the split of the TO and LO modes in polar semiconductor nanowires (Mahan et al., 2003). Indeed, the highly anisotropic shape of the nanowires determines different contribution in the dipolar sums for the components in the cross sectional plane $-\mathrm{x}$ and $\mathrm{y}-$ which are truncated by the finite size, from the one along the nanowire growth axis - z -. Accordingly, the local electric field is modified too. The dispersion relation of the optical phonon can be related to the local spring constant $\left(\omega_{o}^{2}\right)$ and to the local electric field $\left(E_{\mu}\right)$ :

$$
\omega^{2} q_{\mu}=\omega_{o}^{2} q_{\mu}-\frac{e^{*}}{M} E_{\mu}
$$

where $e^{*}$ is the Szigeti charge and M is the reduced mass of the ion pair. The local field can be expressed as:

$$
E_{\mu}=-T_{\mu v}\left[e^{*} q_{v}+\alpha E_{v}\right]
$$

with $T_{\mu \nu}$ the components of the dipole-dipole interaction, $a$ the polarizability of the unit cell and $\alpha E_{v}$ the induced dipole in the same cell from core polarization. The anisotropy in the dipole sums $\left(T_{x x}=T_{y y}=\frac{4 \pi}{6 v_{o}}\right.$ and $\left.T_{z z}=\frac{-4 \pi}{3 v_{o}}\right)$ for a thin wire with $L>>$, with $L$ length and $2 R$ diameter of the wire, results into the anisotropy of the dielectric function, whose tensor has now two different components, $\varepsilon_{x x}=\varepsilon_{y y}$ and $\varepsilon_{z z}$, expressed by: 


$$
\varepsilon_{i j}(\omega)=\varepsilon_{i j}(\infty) \frac{\omega^{2}-\omega_{L j}^{2}}{\omega^{2}-\omega_{T j}^{2}}, \quad j=x, z
$$

If we consider the optical phonons in a nanowire, equations (6) and (7) can be solved considering the uniaxial geometry, thereby obtaining:

$$
\begin{gathered}
\omega_{L z}^{2}=\omega_{L O}^{2}=\omega_{O}^{2}+\omega_{p}^{2} \frac{2\left(\varepsilon_{z}(\infty)+2\right)}{9 \varepsilon_{z}(\infty)} \\
\omega_{T z}^{2}=\omega_{T O}^{2}=\omega_{O}^{2}-\omega_{p}^{2} \frac{\left(\varepsilon_{z}(\infty)+2\right)}{9}
\end{gathered}
$$

with $\varepsilon=1+\frac{4 \pi \alpha / V_{o}}{1-4 \pi \alpha / 3 V_{o}}$ high frequency dielectric constant and $\omega_{p}^{2}=\frac{4 \pi e^{* 2}}{M V_{o}}$ ion plasma frequency, for the $\mathrm{z}$ direction, and

$$
\begin{aligned}
& \omega_{L x}^{2}=\omega_{O}^{2}+\omega_{p}^{2} \frac{\left(\varepsilon_{x}(\infty)+2\right)}{9\left(\varepsilon_{x}(\infty)+1\right)} \\
& \omega_{T x}^{2}=\omega_{O}^{2}+\omega_{p}^{2} \frac{7\left(\varepsilon_{x}(\infty)+2\right)}{9\left(\varepsilon_{x}(\infty)-1\right)}
\end{aligned}
$$

with $\varepsilon_{x}(\infty)=\frac{3 \varepsilon_{z}(\infty)-1}{\varepsilon_{z}(\infty)+1}$ for the other directions. The predicted positions of the triplet arising from the split of the optical phonon due to the nanowire geometry are very close and therefore not always easily distinguishable. For example, in the case of GaAs or GaP nanowires, the $\omega_{L x}$ and $\omega_{T x}$ modes are about $2 \mathrm{~cm}^{-1}$ shifted from the $\omega_{L z}$ mode (Cao et al., 2007). Nevertheless, an indication of the split can be given by the different position of the LO band in the nanowire spectra respect to the bulk. It has been shown that this shape dependence can explain even the occurrence of an angular dependencies of the phonon modes which otherwise would not be expected from the selection rules (Livneh et al., 2006; Fréchette \& Carraro, 2006; Cao et al., 2006).

The reduction in the dimensionality and the presence of edge/boundaries in the crystal can also lead to the appearance/activation in the Raman spectra of inactive Raman modes (silent modes) at the $\Gamma$ point of the Brillouin zone. This is due to the fact that the symmetry is changed by the existence of the edges, which leads to a rearrangement of the lattice structure. This has been especially observed in nanocrystals (Li et al., 2002; Kawashima \& Katagiri, 1999).

Furthermore, there are other size-related phonons appearing when dealing generally with nanostructures, such as the surface optical phonons (SO) and breathing modes. Several works have reported the presence of a further peak in the Raman spectra of semiconductor nanowires or nanoparticles which have been assigned to SO phonons (Gupta et al., 2003a; Shan et al., 2006; Lin et al., 2003; Zeng et al., 2006; Spirkoska et al., 2008). The surface optical phonons are generated at the interface between different materials with different dielectric functions and propagate along the interface. The atoms involved in their propagation are 
those close to the surface, so that the amplitude of the oscillations decays exponentially with the distance from the surface. This mode is activated by a breaking of the translational symmetry of the surface potential, which in the case of the nanowire can be addressed to the presence of roughness, sawtooth faceting on the nanowire sidewall or to a diameter oscillation along the nanowire length.

There are two characteristics which are distinctive of the SO modes and can therefore allow a reliable assignment of the mode: the dependence of the position (1) on the dielectric constant of the medium surrounding the wires and (2) on the diameter (or on the period of the diameter oscillation) of the wires. Indeed, it has already been shown that the SO mode position down shift increasing the dielectric constant of the surrounding optical medium and decreasing the nanowire diameter (Shan et al., 2006; Adu et al., 2006a; Spirkoska et al., 2008). Furthermore, the frequency of the SO modes at the center of the Brillouin zone is located between those of the TO and the LO.

The SO modes dispersion at the interface between a semiconductor and a dielectric material can be calculated imposing the condition:

$$
\varepsilon(\omega)+\varepsilon_{m}=0
$$

with $\varepsilon(\omega)$ the dielectric function of the semiconductor and $\varepsilon_{m}$ the dielectric constant of the medium. In the case of an infinitely long cylinder equation (13) becomes:

$$
\varepsilon(\omega)+\varepsilon_{m} f(q r)=0
$$

where $f(q r)$ is given by

$$
f(q r)=\frac{I_{o}(q r) K_{1}(q r)}{I_{1}(q r) K_{o}(q r)}
$$

With $q$ the phonon wavevector, $r$ the nanowire radius and $I_{i}(q r)$ and $K_{j}(q r)$ the modified Bessel functions. Indeed, the dispersion relation for a SO mode for an infinitely long cylinder can be expressed by:

$$
\omega_{S O}^{2}(q)=\omega_{T O}^{2}+\frac{\tilde{\omega}_{p}^{2}}{\varepsilon_{\infty}+\varepsilon_{m} f(q r)}
$$

with $\omega_{T O}$ the TO mode frequency, $\tilde{\omega}_{p}$ the screened ion plasma frequency given by $\tilde{\omega}_{p}^{2}=\varepsilon_{\infty}\left(\omega_{L O}^{2}-\omega_{T O}^{2}\right), \varepsilon_{\infty}$ the high frequency dielectric constant of the bulk material, $\varepsilon_{m}$ the dielectric constant of the surrounding medium. Equation (16) establishes, therefore, the dependency of the surface phonon energy on the external medium and on the size of the wire, since the position of the surface optical phonon can be related to the dielectric constant of the surrounding medium as well as to the nanowire radius. Furthermore, values of $q$ for the activation of the SO mode can be determined experimentally (Gupta et al., 2003a). Instead, the line width of the surface mode has not been yet well understood.

The effect of the position of the SO modes can be clearly observed by comparing semiconductor nanowires with various diameters. As an example, we show the Raman 
spectra of GaAs nanowires of diameters 160 and $69 \mathrm{~nm}$ in Fig. 6a. For the nanowire with the largest diameter, the SO mode is barely observed as the position is very close to the LO phonon. As expected, the SO mode shifts to lower wavenumbers for smaller diameters, as it can be seen in the spectra obtained for nanowires with an average diameter of $69 \mathrm{~nm}$. The entire trend of the position as a function of the diameter is shown in Fig. $6 \mathrm{~b}$. There, the line indicates what would be expected for nanowires with a circular section-GaAs nanowires exhibit a hexagonal section, which explains the discrepancy with the experimental data-. More details on the experiments can be found elsewhere (Spirkoska et al., 2008).

(a)

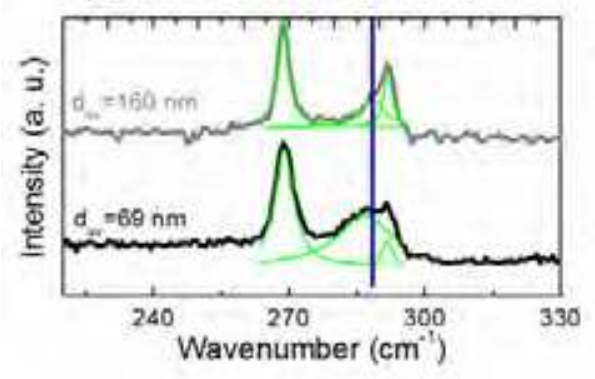

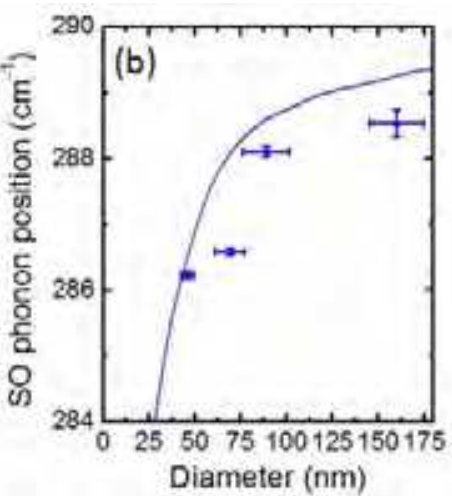

Fig. 6. a) Raman spectra of GaAs nanowire bundles with respectively an average diameter of 160 and $60 \mathrm{~nm}$. The SO mode can be observed on the left of the LO mode b) Evolution of the $\mathrm{SO}$ phonon position as a function of the diameter of the nanowires. The line corresponds to the theoretical values expected for cylindrical GaAs nanowires.

Indeed, it has been proved that the cross section of the nanowires influences the surface mode dispersion (Adu et al., 2006a; Xiong et al., 2006). In these works, a model for nanowire with rectangular cross section has been developed. By setting $z$ as growth direction and defining $L_{i} \quad(i=x, y)$ the edges of the rectangular cross section, the $\mathrm{SO}$ dispersion can be found solving the equations:

$$
\begin{aligned}
& \varepsilon_{n w}(\omega) \tanh \left(\frac{q_{i} L_{i}}{2}\right)+\varepsilon_{m}=0 \\
& \varepsilon_{n w}(\omega) \operatorname{coth}\left(\frac{q_{i} L_{i}}{2}\right)+\varepsilon_{m}=0
\end{aligned}
$$

where $q_{i} \quad(i=x, y)$ is the phonon wavevector of the modes propagating along $\mathrm{x}$ or $\mathrm{y}$, which are the directions affected by the size effects, assuming the wire infinitively long along the $z$ direction. Equation (17a) gives the symmetric mode, while equation (17b) the asymmetric one. Two more conditions have to be fulfilled:

$$
\begin{aligned}
& q_{x}^{2}+q_{y}^{2}=q^{2} \\
& q_{x} L_{x}=q_{x} L_{y}
\end{aligned}
$$


the latter one imposing the same parity to the optical phonon potential in the $x$ and $y$ directions. The symmetric and asymmetric SO phonon dispersion can then be expressed by:

$$
\begin{gathered}
\omega_{\mathrm{SO} S}^{2}(q)=\omega_{T O}^{2} \frac{\varepsilon_{o} \tanh \left(\frac{q_{i} L_{i}}{2}\right)+\varepsilon_{m}}{\varepsilon_{\infty} \tanh \left(\frac{q_{i} L_{i}}{2}\right)+\varepsilon_{m}} \\
\omega_{\mathrm{SO} A}^{2}(q)=\omega_{T O}^{2} \frac{\varepsilon_{o} \operatorname{coth}\left(\frac{q_{i} L_{i}}{2}\right)+\varepsilon_{m}}{\varepsilon_{\infty} \operatorname{coth}\left(\frac{q_{i} L_{i}}{2}\right)+\varepsilon_{m}}
\end{gathered}
$$

The comparison between equations 16 and 19 for the circular and rectangular sections leads to the conclusion that both the shape and size of the cross section have an important influence on the positon of the SO phonon.

Beside the surface modes, it is worth shortly mentioning another mode found in nanowires which cannot be observed in bulk materials: the Radial Breathing Mode (RBM). This mode was first observed in carbon nanotubes, corresponding to the atomic vibration of the carbon atoms in the radial direction. Its frequency was found to be highly dependent on the nanotubes diameter (Alvarez et al., 2000; Jorio et al., 2003; Maultzsch et al., 2005). The same mode has been observed even in semiconductor nanowires (Thonhauser \& Mahan, 2005; Lange et al., 2008), and in both cases the inverse dependence on the nanowire diameter has been found. Assuming the nanowire as an infinitely long isotropic cylinder, the linear elasticity theory furnishes an expression of the RBM:

$$
\omega=\frac{2 \tau_{n}}{d} \sqrt{\frac{E(1-v)}{\rho(1+v)(1-2 v)}}
$$

with v the Poisson's ratio, $\rho$ the nanowire specimen density, $E$ the Young's modulus of the nanowire material and $\tau_{n}$ given by $\sqrt[n]{\tau J_{o}(\tau)}=\sqrt[n]{\frac{(1-2 v)}{(1-v)} J_{1}(\tau)}$ where $J_{i}$ are the Bessel functions. Equation (14) establishes the $1 / d$ dependence of the radial mode frequency, being all the other terms dependent only on the material properties.

\section{Confinement, heating effects and Fano resonance scattering}

\subsection{Phonon quantum confinement in nanowires}

Some of the novel fundamental properties found in nanostructures are related to carrier and phonon confinement (Fischer et al., 2006; De Franceschi et al., 2003; Wanwees et al., 1988; Samuelson et al., 2004; Hu et al., 2007; Shorubalko et al., 2008; Rao et al., 1997; Bawendi et al. 1990; Lehmann et al., 1991). Confinement is usually correlated with tailoring novel physical properties, often giving rise to novel applications (Faist et al., 1994; Somers et al., 2008; Steckel et al., 2003). Raman spectroscopy is an ideal and relatively straightforward technique to test quantum confinement. Moreover, it can be realized under many extreme and non- 
extreme conditions, leading to an ideal technique for the investigation of processes in matter at low/high temperatures and/or high pressures (Iwasa et al., 2004; Kim et al., 1996; Wright et al., 1997; Weinstein et al., 1975; Congeduti et al., 2001). Indeed, phonon scattering in crystals of small dimension leads to a redshift and broadening of the first order Raman line. This is due to the relaxation of the $\mathrm{q}=0$ selection rule when the volume objects becomes of the order of few phonon wavelengths. For nanoscale object such as nanocrystals or nanowires, the exact shape of the Raman peak becomes a convolution of the dispersion relation of phonons in the material (Richter et al., 1981; Campbell et al., 1986). Such effect was initially observed in nanocrystals and more recently in nanowires (Fauchet et al., 1988; Adu et al., 2006a; Jalilian et al., 2006; Fukata et al., 2006). In the particular case of nanowires, the confinement occurs in the diameter direction. It has been predicted and experimentally confirmed that the Raman scattering intensity $I_{S}(\omega, d)$ for a diameter $d$ at a photon frequency $\omega$ relative to the laser frequency is given by (Campbell et al., 1986):

$$
I_{S}(\omega, d)=I_{O} \cdot \int_{0}^{1} 2 \pi q_{\perp} \cdot d q_{\perp} \cdot \frac{\left|C\left(q_{\perp}\right)\right|^{2}}{\left(\omega-\omega_{O}\left(q_{\perp}, T\right)\right)^{2}+\Gamma(T)^{2} / 4}
$$

Where $\left|C\left(q_{\perp}\right)\right|^{2} \prec \exp \left(-\alpha a_{O} / \sqrt{2} \cdot q_{\perp} d\right)$ is the confinement function, $a_{0}$ the lattice constant of the material, $\alpha$ a material dependent constant, $q_{\perp}$ is the phonon wave vector in perpendicular to the nanowire axis, $1 / \Gamma$ the phonon lifetime. In the case where Raman spectroscopy is realized on nanowire pads ensembles, it is essential to take into account of the nanowire diameter distribution for the exact modeling of the experimental curves (Adu et al., 2005). These observations extend to most of materials systems from silicon, germanium, zinc oxide, gallium phosphide, zinc sulfide... Equation (18) suggests that reducing the diameter of a cylindrical nanowire results in a redshift (towards lower frequencies) and a broadening of the Raman line. The exact shape of the spectrum is given by the equation and it would vary for other types of geometry, such as spherical or cubical nanoparticles or cylindrical or prismatic nanowires. In the case of silicon, a maximum shift of $8 \mathrm{~cm}^{-1}$ is observed for $4 \mathrm{~nm}$ nanowires (Adu et al., 2005).

In the following, we present an example of spatially resolved Raman spectroscopy measurements, indicating regions of the nanowire where the functional material achieves nanometer dimensions. Thereby, it helps to predict if it will be possible to obtain functional electronic devices with the nanowires. The samples consisted of germanium nanowires grown by chemical vapor deposition by using indium as a catalyst, the details reported elsewhere (Xiang et al., 2009). Structural analysis of the nanowires evidenced that they consisted in a crystalline core, surrounded by an amorphous shell, as shown in Fig.7a. Interestingly, it was shown that the crystalline core was not continuous along the nanowire and that it could shrink down to $\sim 10 \mathrm{~nm}$ in diameter -see Fig. $7 \mathrm{~b}$-. The shrinking of the core poses many problems if these nanowires are to be used for electronic devices, as they will inevitably be short-circuited. A non-destructive diagnosis such as Raman can provide the information on what regions of the nanowire can be used for the devices. For that, it is necessary to realize scanning Raman spectroscopy measurements along the whole length of the nanowire. An example is shown in Fig. 7c. There, $100 \mathrm{~nm}$ spaced Raman spectra along 
an $86 \mathrm{~nm}$ wide germanium nanowire are shown -the diameter was obtained by measuring the height in an Atomic Force Microscope scan-. In the measurements, only the peak corresponding to the TO/LO phonon mode of crystalline germanium is observed. In the small diameter nanowires we do not observe the contribution of the amorphous band. This could be due to various effects: 1) due to the small diameter, the fraction of amorphous germanium is significantly smaller than for a nanowire with larger diameter 2) the density of the amorphous shell is smaller than that of the crystalline core. For reference, we have plotted the position of the unstrained germanium. Along the $2 \mu \mathrm{m}$ of the scan, a recurrent shift towards lower frequencies is observed. The shift can be attributed to the phonon confinement in the core of the nanowire. The data fit well with the model in which the nanowire is assumed to have a spherical nancrystal shaper shape. According to this, the observed downshift of $6 \mathrm{~cm}^{-1}$ corresponds to a diameter smaller than $30 \mathrm{~nm}$. The spatially resolved Raman scattering measurements indicate a variation of the core diameter along the nanowire, which are in agreement with the transmission electron micrographs realized.

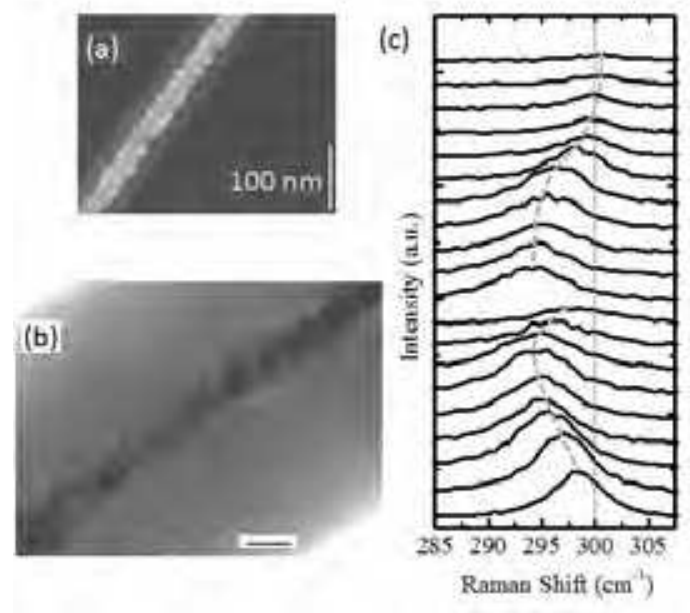

Fig. 7. a) Scanning TEM annular dark field micrograph obtained in one part of a 10 micron long germanium nanowire, showing a $40 \mathrm{~nm}$ multi-crystalline core, capped with a $21 \mathrm{~nm}$ thick amorphous layer $b$ ) Bright field TEM micrograph of a part of a germanium nanowire where the crystalline core is $5 \mathrm{~nm}$. The amorphous shell is $40 \mathrm{~nm}$ thick,

c) Waterfall plot of Raman spectra taken every $100 \mathrm{~nm}$ of a thin nanowire. As a guide to the eye, the light grey line indicates the position of the TO/LO unstrainedGe mode, and the thick dashed line indicates the position of the Raman mode in the nanowire.

Relatively recent studies have shown that one should be very precautious in the analysis of quantum confinement measurements (Campbell et al., 1986; Fauchet et al., 1988). Indeed, note that in the eq. (18) the phonon frequency $\omega_{O}\left(q_{\perp}\right)$ and the phonon lifetime $1 / \Gamma$ are a function of temperature. One should also note that the radiation power density incident on the nanowire increases dramatically for small diameter nanowires. Indeed, for equal diameter spot the volume of sample illuminated is proportional to $d^{2}$. As a consequence, the power density received by the sample is proportional to $1 / \mathrm{d}^{2}$. This immediately points 
out the possibility of inevitable heating in the case of extremely small diameter nanowires (the ones expected to exhibit quantum confinement). Additionally, high excitation power densities create a high density of free carriers. This is especially true for indirect bandgap semiconductors such as silicon which exhibit long recombination times. The carriers can also interfere with the phonons giving rise to Fano phenomenon and create an asymmetric line shape (Compaan et al., 1985). In the next sections we discuss these effects on the shape of the Raman spectra.

\subsection{Heating effects during Raman spectroscopy measurements}

A typical effect of laser irradiation on nanoscale samples is heating. This effect is amplified due to the relative increase in the power density, consequence of the sample geometry. It is also a consequence of the lower thermal conductivity of nanowires and of thermal insulation between the nanoscale object -e.g. nanowire- and the substrate ( $\mathrm{Li}$ et al., 2003). The usual way to increase the temperature of samples during Raman spectroscopy measurements is to increase the incident irradiation power. An example of the effect on heating on the Raman spectrum is shown in Fig. 8. There, Raman spectroscopy measurements of a single GaAs nanowire as a function of the incident power density are shown. Clearly, both the TO and the LO modes become increasingly asymmetric as the incident power density is increased. A shift of the peak position towards lower wavenumbers is also clear. Between the two effects, the asymmetric broadening is the first one that arises. This tendency can be clearly seen in the graph of Fig. 8b, where the evolution of the peak positon and FWHM is shown for each excitation power.
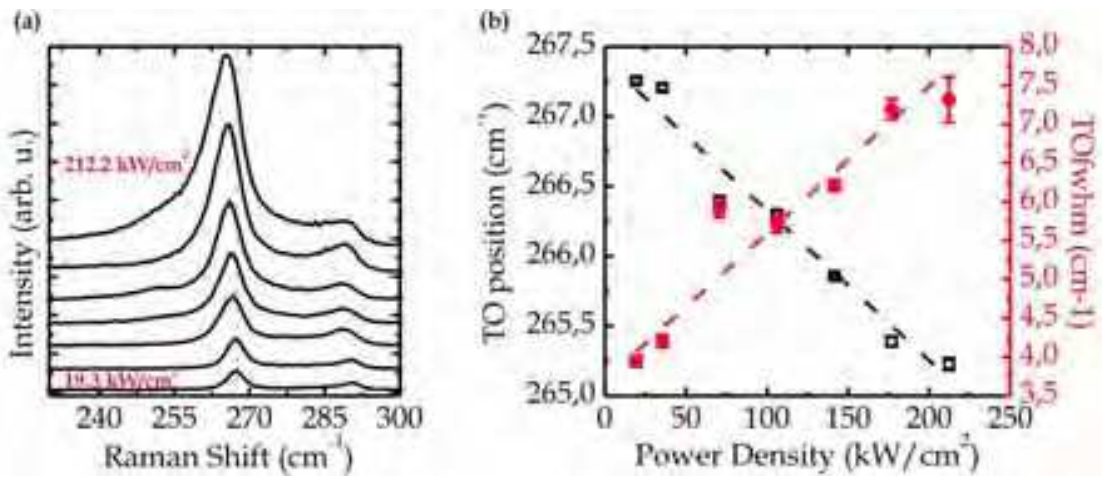

Fig. 8. Raman spectra of zinc-blende GaAs nanowire bundles collected increasing the power density from 19.3 till $212.2 \mathrm{~kW} / \mathrm{cm}^{2}$. b) Position and FWHM of the TO mode of the spectra shown in a), as a function of the power density. The dashed lines are linear fit to the data.

The temperature of the nanowire $T$ upon laser heating is usually estimated by calculating the ratio of the integrated intensity between the Stokes and Anti-Stokes peaks $-\boldsymbol{I}_{S}$ and $\boldsymbol{I}_{A S^{-}}$at the phonon frequency $\omega_{o}$, which is (Balkanski et al., 1983):

$$
\frac{I_{S}}{I_{A S}}=\exp \left(\frac{\hbar \omega_{o}}{K T}\right)
$$

In the case of homogeneous heating of a material, the effect of temperature on the Raman line shape is due to: 1) the decrease in the phonon frequencies $\omega_{o}$ because of thermal 
expansion and 2) to the increase in the inverse optical phonon lifetime $1 / \Gamma$ for $q=0$ (Balkanski et al., 1983). For a uniform heating, the effect of temperature increase should homogeneously broaden and shift the Raman line. However, experimentally an asymmetric broadening is always observed (Jalilian et al., 2006; Piscanec et al., 2003). This observation can only be explained by the existence of temperature gradients along the nanowire. Indeed, one should consider the laser intensity distribution $I(z)=I_{0} e^{(-z / a)}-$ Gaussian- and the induced temperature response $T(z)$ due to the thermal conductivity and capacity of the nanowire. Then, equation 18 is transformed in the following expression for the description of the line shape function (Adu et al. 2006b):

$$
I(\omega)=\int_{-c}^{c} d z I_{o} e^{(-z / a)^{2}} \int_{0}^{1} 2 \pi q_{\perp} d q_{\perp} \cdot \frac{\left|C\left(q_{\perp}\right)\right|^{2}}{\left(\omega-\omega_{O}\left(q_{\perp}, T\right)\right)^{2}+\Gamma(T)^{2} / 4}
$$

It has been demonstrated that the use of this equation for the fitting of the Raman spectra is essential to decouple the effect of thermal heating and confinement (Adu et al., 2006a, Adu et al., 2006b).

An additional consequence of the heating may be structural phase transformation. Indeed, other crystalline structures may be rendered stables at higher temperatures. This phenomenon may be accentuated by the geometry of nanoscale objects, which exhibit a much larger surface-to-volume ratio (Wickham et al., 2000). As an example, we have investigated the evolution of Raman spectra of silicon nanowires with hexagonal structure after heating them with the excitation laser -the measurements were realized at room temperature-. The hexagonal or lonsdaleite structure is a metastable phase of silicon under normal conditions, also denominated as Si-IV. It has been reported by several authors in the form of nanowires (Fontcuberta i Morral et al., 2007; Lopez et al., 2009; Arbiol et al.; 2008). Being Si-IV a metastable phase, it is expected that it may transform into diamond structure (Si-I) upon heating. In Fig. 9, the spectra of an ensemble of silicon nanowires exhibiting the Si-IV phase is shown. The Raman spectra after three annealing treatments of 200s at 60, 100 and $140 \mathrm{~kW} / \mathrm{cm}^{2}$ are also shown. The Raman spectra at the end of the irradiation are fitted to obtain the temperature, which corresponds to 200, 440 and $600^{\circ} \mathrm{C}$. After the first

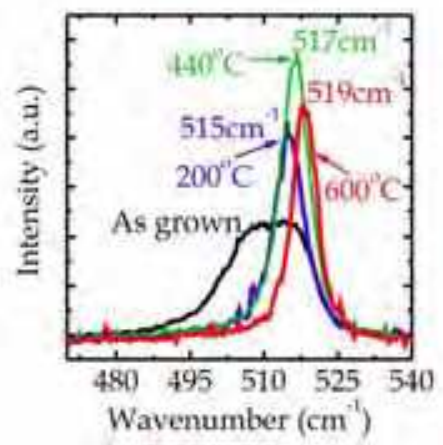

Fig. 9. Raman spectra of Si-IV nanowire bundles as grown and after heating them by illumination with the Raman objective at temperatures of 1) $200^{\circ} \mathrm{C}$ 2) $440^{\circ} \mathrm{C}$ and 3) $600^{\circ} \mathrm{C}$. 
treatment, the Raman peak related to the Si-IV phase disappears completely, and a new unique peak at $515 \mathrm{~cm}^{-1}$ appears. By successive annealing, the peak shifts to 517 and $519 \mathrm{~cm}^{-1}$. This indicates that the sample structure continues to consist in diamond silicon. Additionally, the correlation length of the phonons increases, in agreement with the TEM results showing an improvement in the crystalline structure -increase in grain size- (Prades et al., 2007).

\subsection{Fano resonant scattering}

Finally, we address another effect resulting from the use of high excitation power densities in Raman scattering experiment. High excitation power densities create a high density of free carriers, which can interfere with the phonon scattering. Fano interference in Raman scattering has been extensively studied in highly doped bulk silicon samples (Belitsky et al., 1997; Arya et al., 1979). It results in an asymmetric line shape of the first order phonon Raman peak, following the equation (Madidson et al., 2002):

$$
I(\omega)=C+\sigma_{o} \frac{(q+\varepsilon)^{2}}{1+\varepsilon^{2}} \quad \varepsilon=\frac{\omega-\omega_{o}}{\Gamma}
$$

Where $\omega$ is the scattered photon energy, $\omega_{o}$ and $\Gamma$ are respectively the resonance frequency and width, and $\sigma_{o}$ and $C$ are constants. The influence of Fano scattering on the Raman spectra is determined by $\boldsymbol{q}$, the asymmetry parameter. In fact, it has been generally found in bulk silicon that $1 / q$ is proportional to the free carrier concentration. The curve becomes Lorenzian for $q \rightarrow \infty$ and the asymmetry increases as the value gets smaller. In Fig. 10, we have plotted the shape of the Raman peak of germanium for different values of $\boldsymbol{q}$. There, it is clear that values of $\mathrm{q}$ of 10 start to be enough to create an asymmetry in the Raman spectrum. Experimentally, values of $\mathrm{q}$ between 35 and 4 have been measured for highly doped p-type bulk silicon samples (Madidson et al., 2002). In the case of undoped silicon nanowires, values of 8 and 17 have been reported (Gupta et al., 2003b). These studies have demonstrated that taking into account the effect of Fano interference, when fitting the measured Raman spectra. Indeed, Raman scattering of small diameter nanowires is not a straightforward measurement. Effects like quantum confinement, diameter distribution, inhomogeneous heating and Fano interference have to be taken into account correctly for the accurate interpretation.

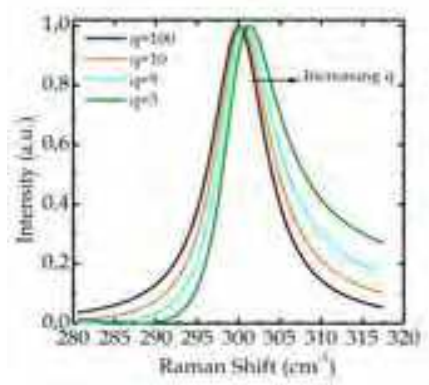

Fig. 10. Illustration of the effect of decreasing $q$ in the asymmetry of one phonon Raman spectra of germanium -calculations following eq. 21). 


\section{Existence of different crystallographic phases in a nanowire: Study of GaAs nanowires with wurtzite/zinc-blende structures}

Most of the binary octet semiconductors such as $\mathrm{GaN}$ and $\mathrm{SiC}$ present either zinc-blende or wurtzite structure, which correspond to the cubic and hexagonal structure with two atoms per basis. From the crystallographic point, the two structures differ only in the stacking periodicity of the atomic layers along the c-axis of the hexagonal structure. The stacking sequence is 'abcabc' for the cubic structure and 'abab' for the hexagonal one, as shown in Fig. 11 (Park et al., 1994).
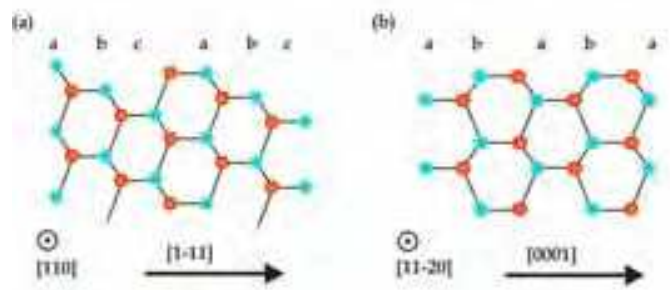

Fig. 11. Schematic drawing of the atomic arrangement in zinc-blende (a) and wurtzite (b) structures. The arrows indicate the [1-11] and the [0001] nanowire growth axes, respectively.

The spectroscopic, electronic and thermal conductivity properties of these two structures and of their polytypisms can be very different (Yeh et al., 1992). Especially interesting are structures formed by the two crystallographic phases, a sort of homo-heterostructure, which exhibit novel optical and electronic properties (Spirkoska et al., 2009). Controlled reproduction of polytypisms in materials give new degrees of freedom in the realization of electronic devices and in the structural bandgap engineering (Raffy et al., 2002; Algra et al., 2008; Mishra et al., 2007; Arbiol et al. 2009).

The different stacking order of the planes implies different symmetry groups. This, together with the slightly different lattice parameter should lead to different vibrational properties. Nevertheless - as it has been shown in the case of $\mathrm{GaN}, \mathrm{SiC}$ and $\mathrm{Si}$ - the phonon dispersion of hexagonal structure can be deduced with good accuracy from the phonon dispersion of the cubic one by just considering the different stacking of the ' $a b c$ ' and 'ab' layers (Harima, 2002; Loudon, 2001; Kobliska \& Solin, 1973). The phonon dispersion of the cubic structure along the [111] direction corresponds to the $\Gamma \rightarrow \mathbf{L}$ direction in the Brillouin zone. For clarity, we remind that the $\mathrm{c}$ axis of the hexagonal structure can be indexed in the 4 index Miller notation as [0001], and it is equivalent to the [111] axis of the cubic structure. The unit cell length along the [0001] axis of the hexagonal structure is double than that of the cubic structure along the [111] direction, since they correspond to the width of two and one bilayer, respectively. Consequently, the phonon dispersion of the hexagonal structure along the [0001] axis can be approximated by folding the one of the cubic structure along the [111] axis, as shown in Fig. 12 for the cases of GaN, GaAs and Si (Harima, 2002; Zardo, 2009b; Giannozzi, 1991).

As a consequence of the folding, the phonon modes at the $L$ point are taken back at the $\Gamma$ point of the Brillouin zone, giving rise to four new modes. As an example, in the case of GaAs we have the appearance of the $E_{2}$ and $B_{1}$ modes in the optical branches. In backscattering geometry Raman spectroscopy only the $\mathrm{E}_{2}$ mode can be observed and it should be located at lower frequencies than the $\mathrm{E}_{1}(\mathrm{TO})$ mode. For silicon, a new optical branch appears down from the degenerate $\mathrm{TO} / \mathrm{LO}$ one. One expects to observe a novel vibrational mode around $\sim 500 \mathrm{~cm}^{-1}, 20 \mathrm{~cm}^{-1}$ below the $q=0 \mathrm{TO} / \mathrm{LO}$ mode. 

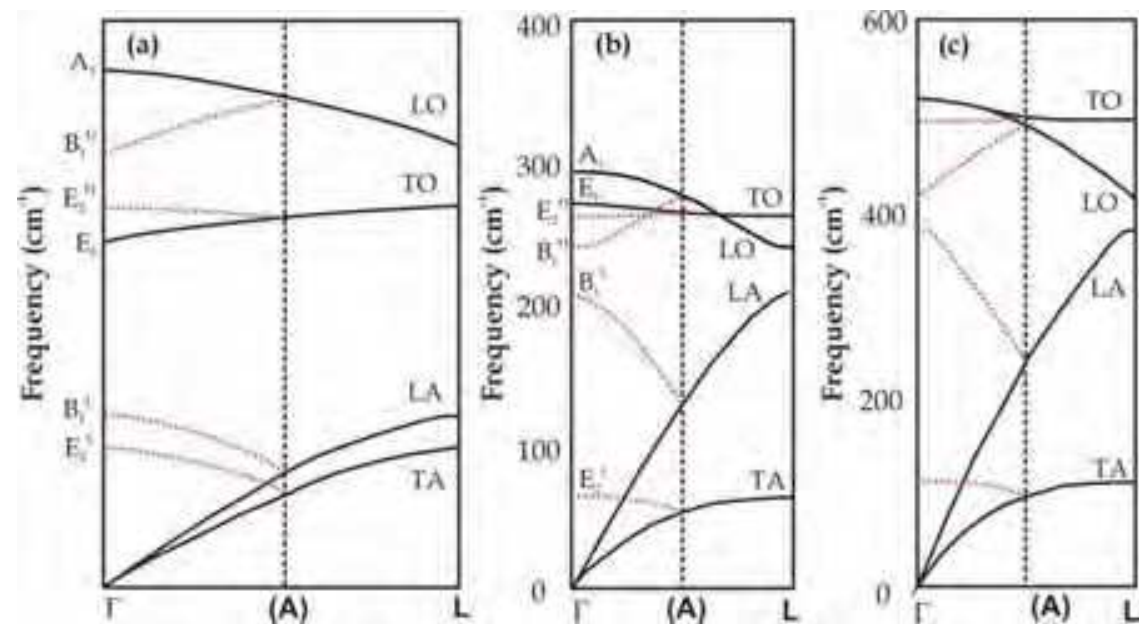

Fig. 12. Schematic representation of the phonon dispersion in GaN (a), GaAs (b) and Si (c). Phonon branches along [111] in the zinc-blende structure are folded to approximate those of wurtzite structure along [0001].

In this context, even the incidence of stacking faults and twins in nanowires gains attention and it is currently under deep investigation (Bandet et al., 2002; Lopez et al., 2009; Algra et al., 2008; Caroff et a., 2009; Zardo et al., 2009a; Conesa-Boj et al., 2009; Arbiol et al., 2009; Spirkoska et al., 2009). Indeed, the atomic stacking can be altered locally from a rotationally twin plane, so that when it occurs in a cubic nanowire gives rise to the occurrence of a monolayer of the hexagonal phase (Arbiol et al., 2009). Furthermore, twins can also cross or exist in high density, resulting into the formation of different structures, localized superstructures or heterostructure phase domains. For example, twinning superlattices are formed whenever twins occur with a certain periodicity. Additionally, the intersection of transversal and lateral twins (twins respectively along or with an angle with the growth axis) can lead to the formation of nanoscale domains with diamond hexagonal phase in the typical silicon cubic structure (Conesa-Boj et al., 2009). As already mentioned above, one should keep in mind that even their polytypisms can have very different physical properties from the pure crystalline phases (Lopez et al., 2009). As it will be shown in the following, Raman spectroscopy is a versatile technique that helps identify materials and areas in the materials with different crystal structures and/or polytypisms. The correlation with Transmission Electron Microscopy measurements can sustain and complement the information.

As an example, we show the case of GaAs nanowires with crystalline structures not stable in the bulk. The stable crystal structure for bulk GaAs is the zinc-blende. However, it has been shown GaAs nanowires can crystallize in the wurtzite structure, as shown in Fig. 13.

Fig. 14 contains an intensity map of the polarization dependent Raman spectra measured with a spacing of $100 \mathrm{~nm}$ along the nanowire. The incident and analyzed polarization are parallel respect to each other, and both perpendicular (Fig.14a) or parallel (Fig. 14b) to the nanowire growth axis $\mathrm{z}$. 


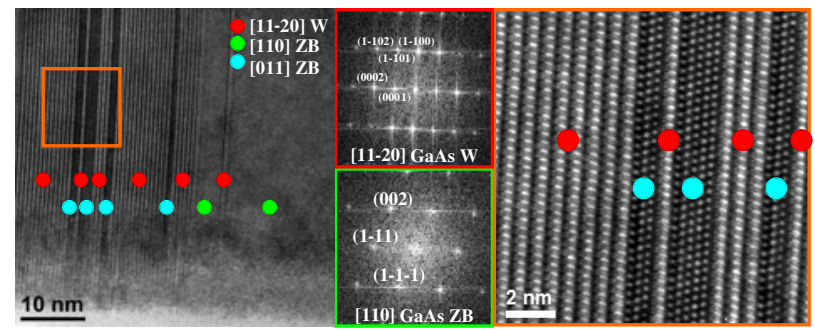

Fig. 13. HRTEM micrographs and power spectra analyses corresponding to GaAs NWs from a sample showing high content of Wurtzite and ZB regions with few monolayers.
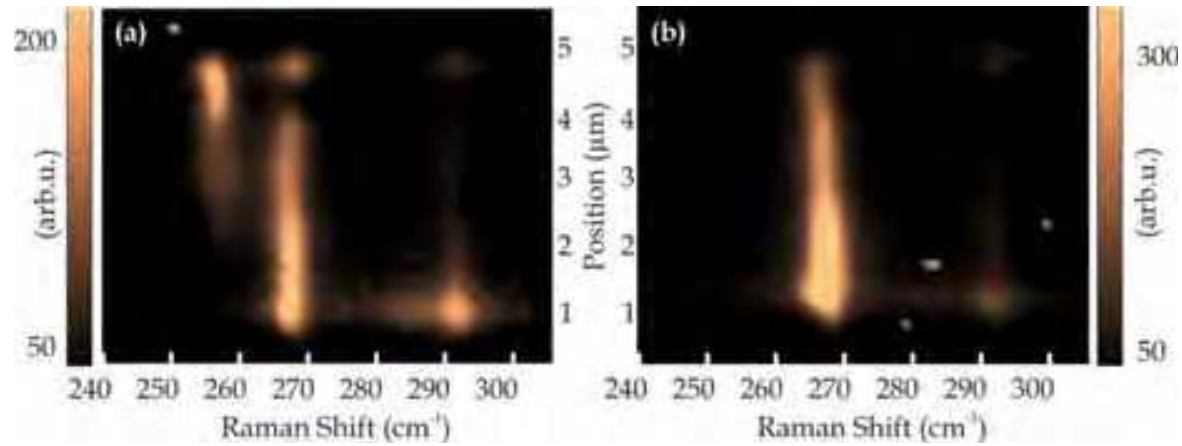

Fig. 14. Color plots showing polarized Raman scans from a nanowire consisting of $30 \%$ of wurtzite structure, obtained using different polarization direction of the incident light: a) Perpendicularly polarized Raman scan from perpendicularly polarized incident light: $x(y, y) \bar{x}$ b) parallel polarized Raman scan from parallel polarized incident light: $x(z, z) \bar{x}$.

The $\mathrm{E}_{1} \mathrm{H}$ (TO) mode is observable for both polarization configurations at $266.7 \mathrm{~cm}^{-1}$, as expected for GaAs nanowire. When the polarization of the incident light is perpendicular to the nanowire axis, a further peak appears. This peak is positioned at about $256 \mathrm{~cm}^{-1}$, which corresponds to the $\mathrm{E}_{2} \mathrm{H}$ (TO) mode from the wurtzite GaAs phase, as a result of the folding of the $\mathrm{E}_{1}$ (TO) branch of the phonon dispersion in the zinc blende structure, as illustrated above. The $\mathrm{E}_{2} \mathrm{H}$ (TO) mode intensity is higher at one end of the nanowire and decreases towards the middle, in good agreement with the percentage of wurtzite phase in the nanowire. Furthermore, in confirmation of the assignment of this peak to the $\mathrm{E}_{2} \mathrm{H}(\mathrm{TO})$ mode of the wurtzite structure, its dependence on the polarization of the excitation follows the Raman selection rules (see Fig. 5).

Another interesting feature of the measurements presented in Fig. 14 is the presence of the $\mathrm{A}_{1}(\mathrm{LO})$ mode. Even though the $\mathrm{A}_{1}(\mathrm{LO})$ mode is not allowed for the backscattering configuration on $\{110\}$ family surfaces, to which the nanowires side facets belong, it is weakly present at $290.9 \mathrm{~cm}^{-1}$ at one end of the nanowire. Its presence is related to the occurrence of highly dense twins in the zinc blende crystal structure, which cause that the facets of the nanowire are not of the family $\{110\}$ anymore, but $\{111\}$. The $A_{1}$ (LO) mode is allowed for backscattering from $\{111\}$. The small $\{111\}$ faceting at the end of the nanowire can explain the increased intensity of the $\mathrm{A}_{1}(\mathrm{LO})$ phonon mode. 
As a further example, we present Raman spectroscopy measurements on nanowires with a relatively high density of twins. Indeed, Raman spectroscopy is extremely sensitive even to structural defects such as the presence of dense stacking faults or twins (Lopez, 2009). Fig. 15 shows Raman spectra from silicon nanowires grown using Indium as catalyst. The nanowires present the [112] growth direction with very high density of twin defects along the $\{111\}$ planes and consequent formation of hexagonal domain. In addition to the peak related to the TO/LO phonon, the presence of an additional peak at about $495 \mathrm{~cm}^{-1} \mathrm{can}$ be observed (Prades et al. 2007). The existence of this peak has been explained by the presence of the highly twinned domains and hexagonal phase in nanowires (Kikkawa et al., 2005; Fontcuberta i Morral et al., 2007; Prades et al., 2007; Algra et al., 2008). The position of the band at $495 \mathrm{~cm}^{-1}$ coincides with the energy with the zone boundary of the phonon dispersion of silicon in the L point.

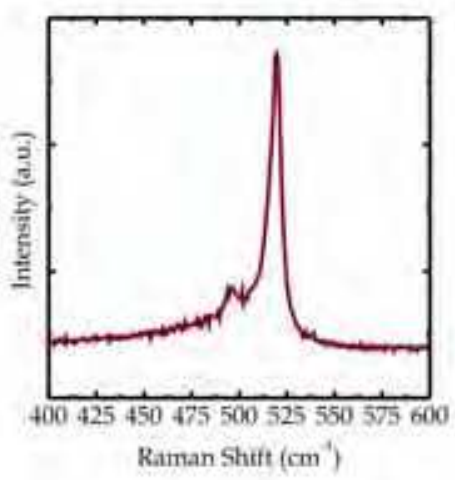

Fig. 15. Raman spectra of indium catalyzed silicon nanowires presenting high density of twins defects. Beside the TO/LO degenerate peak at $520 \mathrm{~cm}^{-1}$, another peak at about $495 \mathrm{~cm}^{-1}$ appears.

\section{Conclusions}

We have presented the fundamentals for understanding Raman scattering on semiconductor nanowires. The basic physical principles of the specific phenomena related to the nanowire nature were presented. We have developed the theory and presented some experimental data on novel phenomena such as inhomogeneous heating, quantum confinement, Fano effect, the existence of surface and breathing modes and the existence of novel crystalline phases.

\section{Acknowledgements}

We thank the courtesy of Dance Spirkoska and J. Daniel Prades for lending us their raw data for the manuscript. Jordi Arbiol, Sonia Conesa-Boj, Francesca Peiro, Sonia Estradé and Joan Ramon Morante are greatly acknowledged for the TEM measurements and analysis. Max Bichler, Emanuele Uccelli, Sara Yazji, Norman Hauke, Ying Xiang and Mark Brongersma are kindly acknowledged for their precious technical help and fabrication of samples. We also 
greatly thank funding from the Marie Curie Excellence Grant 'SENFED', the DFG excellence initiative Nanosystems Initiative Munich and the California-Baviera cooperation program Bacatec.

\section{References}

Abstreiter G. \& Ploog K. (1979). Inelastic light-scattering from a quasi-2-dimenstional electron system in GaAs-AlxGa1-xAs heterojunctions. Phys. Rev. Lett. 42, 1308-11, ISSN: 0031-9007

Abstreiter G., Schittenhelm P., Engel C., Silveira E., Zrenner A., Meertens D. \& Jager W., (1996). Growth and characterization of self-assembled Ge-rich islands on SiSemicond. Sci. Technol. 11, 1521-28, ISSN: 0268-1242

Adu K.W., Gutierrez H.R., Kim U.J., Sumanasekera G.U. \& Eklund P.C. (2005). Confined phonons in Si nanowires. Nano Lett. 5, 409 , ISSN: 1530-6984

Adu K.W., Xiong Q., Gutierrez H.R., Chen G. \& Eklund P.C. (2006a). Raman scattering as a probe of phonon confinement and surface optical modes in semiconducting nanowires. Appl. Phys. A 85, 287, ISSN: 0947-8396

Adu K.W., Gutierrez H. R., Kim U.J. \& Eklund P.C., (2006b). Inhomogeneous laser heating and phonon confinement in silicon nanowires: A micro-Raman scattering study Phys. Rev. B 73, 155333, ISSN: 1098-0121

Algra R.E., Verheijen M.A., Borgström M.T., Feiner L.F., Immink G., van Enckevort W.J.P., Vlieg E. \& Bakkers E.P.A.M. (2008). Twinning superlattice in indium phosphide nanowires. Nature, 456, (Nov 2008) 369-372, ISSN: 0028-0836

Alvarez L., Righi A., Guillard T., Rols S., Anglaret E., Laplaze D. \& Sauvajol J. (2000). Resonant Raman study of the structure and electronic properties of single-wall carbon nanotubes. Chem. Phys. Lett., 316, (Jan 2000) 186-190, ISSN: 0009-2614

Anastassakis E. (1997). Angular dispersion of optical phonon frequencies in strained cubic crystals el Appl. Phys., 82, (Apr 1997) 1582, ISSN: 0021-8979

Arbiol J., Fontcuberta i Morral A., Estrade S., Peiro F., Kalache B., Roca i Cabarrocas P. \& Morante J.R. (2008). Influence of the (111) twinning on the formation of diamond cubic/diamond hexagonal heterostructures in Cu-catalyzed Si nanowire. I Appl. Phys. 104, (Sept 2008) 064312, ISSN: 0021-8979

Arbiol J., Estradé S., Prades J.D., Cirera A., Furtmayr F., Stark C., Laufer A., Stutzmann M., Eickhoff M., Gass M.H., Bleloch A.L., Peiró F. \& Morante J.R. (2009). Triple-twin domains in $\mathrm{Mg}$ doped $\mathrm{GaN}$ wurtzite nanowires: structural and electronic properties of this zinc-blende-like stacking. Nanotechnology, 20, 14, (Apr 2009) 145704, ISSN: 0975-4484

Arya K., Kanehisa M., Jouanne M., Jain K. \& Balkanski M., (1979). Pseudopotential calculation of the discrete-continuum interference in p-Si Raman spectra. I Phys. C 12, 18, 3843-8, ISSN: 0022-3719

Balkanski M., Wallis R.F. \& Haro E. (1983). Anharmonic effects in light-scattering due to optical phonons in silicon Phys. Rev. B 28, 4, 1928-34, ISSN: 1098-0121

Bandet J., Despax B. \& Caumont M. (2002). Vibrational and electronic properties of stabilized wurtzite-like silicon. J. Phys. D: Appl. Phys., 35, (Jan 2002) 234-9, ISSN: 0022-3727 
Baumgartner M. \& Abstreiter G. (1984). Interaction between electronic and phonon Ramanscattering in hole space-charge layers on silicon. Surf. Sci., 142, 357-60, ISSN: 00396028, ISSN: 0031-9007

Bawendi M.G., Wilson W.L., Rothberg L., Carroll P.J., Jedju T.M., Steigerwald M.L. \& Brus . (1990). Electronic-structure and photoexcited-carrier dynamics in nanometer-size CdSe clusters. Phys. Rev. Lett., 65, (Sep 1990) 1623-26, ISSN: 0031-9007

Belitsky V.I., Cantarero A., Cardona M., Trallero-Giner G. \& Pavlov S. (1997). Feynman diagrams and Fano interference in light scattering from doped semiconductors. $C$ Phys. Condens. Matter. 9, (Jul 1997) 5965, ISSN: 0953-8984

Cao L.Y., Laim L., Valenzuela P.D., Nabet B. \& Spanier J.E. (2007). On the Raman scattering from semiconducting nanowires. \& Raman. Spectrosc. 38, (Jun 2007) 697-703, ISSN: 0377-0486

Cao L.Y., Nabet B. \& Spanier J.E. (2006). Enhanced raman scattering from individual semiconductor nanocones and nanowires. Phys. Rev. Lett., 96, (Apr 2006) 157402-5, ISSN: 0031-9007

Cao L., White J. S., Park J. S., Schuller J. A., Clemens B. M. \& Brongersma M. L. (2009). Engineering light absorption in semiconductor nanowire devices. Nat. Mat., 8, (Aug 2009) 643-647, ISSN: 1476-1122

Campbell I.H. \& Fauchet P.M. (1986). The effects of microcrystal size and shape on the one phonon Raman-spectra of crystalline semiconductors. Solid State Commun., 58, (June 1986) 739-41, ISSN: 0038-1098

Caroff P., Dick K.A., Johansson J., Messing M.E., Deppert K. \& Samuelson L. (2009). Controlled polytypic and twin-plane superlattices in III-V nanowires. Nat. Nanotechnology, 4, (Jan 2009) 50-55, ISSN: 0957-4484

Compaan A., Lee M.C. \& Trott G.J., (1985). Phonon populations by nanosecond pulsed Raman-scattering in Si. Phys. Rev. B 32, 10, 6731-41, ISSN: 0163-1829

Conesa-Boj S., Zardo I., Estradé S., Wei L., Alet P.J., Roca i Cabarrocas P., Morante J.R., Peiró F., Fontcuberta i Morral A. \& Arbiol J. (2009).Gallium catalyzed Silicon Nanowires: formation of lateral and transversal twin domains. Submitted to Crystal Growth \& Design (2009)

Congeduti A., Postorino P., Caramagno E., Nardone M., Kumar A. \& Sarma D.D. (2001). Anomalous high pressure dependence of the Jahn-Teller phonon in La0.75Ca0.25MnO3. Phys. Rev. Lett., 86, (Feb 2001) 1251-4, ISSN: 0031-9007

De Franceschi S., van Dam J.A., Bakkers E.P.A.M., Feiner L.F., Gurevich L. \& Kouwenhoven L.P. (2003). Single-electron tunneling in InP nanowires. Appl. Phys. Lett., 83, (Jul 2004) 344-6, ISSN: 0003-6951

Duesberg G.S., Loa I., Burghard M., Syassen K. \& S Roth S. (2000). Polarized Raman Spectroscopy on Isolated Single-Wall Carbon Nanotubes. Phys. Rev. Lett. 85, 25, (Dec 2000) 5437-9, ISSN: 0031-9007

Faist J., Capasso F., Sivco D.L, Sirtori C., Hutchinson A.L. \& Cho A.Y. (1994). Quantum cascade laser. Science, 264 , (Apr 1994) 553-6, ISSN: 0036-8075

Fauchet P.M. \& Campbell I.H. (1988). Raman-spectroscopy of low-dimensional semiconductors, Crc. Critical reviews in solid state and materials sciences 14, S79-101, ISSN: 0161-1593 
Fischer S.F., Apetrii G., Kunze U., Schuh D. \& Abstreiter G. (2006). Energy spectroscopy of controlled coupled quantum-wire states. Nat. Phys., 2, (Feb 2006) 91-6, ISSN: 17452473

Fontcuberta i Morral A., Arbiol J., Prades J.D., Cirera A. \& Morante J.R., (2007). Synthesis of silicon Nanowires with Wurtzite Crystalline Structure by Using Standard Chemical Vapor Deposition. Adv. Mater., 19, (May 2007) 1347-1351, ISSN: 0935-9648

Fukata N., Oshima T., Okada N., Murakami K., Kizuka T., Tsurui T. \& Ito S. (2006). Phonon confinement and self-limiting oxidation effect of silicon nanowires synthesized by laser ablation. I Appl. Phys., 100, (Jul 2006) 014311, ISSN: 0021-8979

Fréchette J. \& Carraro C. (2006). Diameter-dependent modulation and polarization anisotropy in Raman scattering from individual nanowires. Phys. Rev. B, 74, (Oct 2006) 2161404, ISSN: 1098-0121

Giannozzi P., de Gironcoli S., Pavone P. \& Baroni S., (1991). Ab initio calculation of phonon dispersions in semiconductors. Phys. Rev. B, 43, (March 1992) 7231-7242, ISSN: 01631829

Goni A.R. A. Pinczuk, Weiner J.S., Calleja J.M., Dennis B.S., Pfeiffer L.N. \& West K.W., (1991). One-dimensional plasmon dispersion and dispersionless intersubband excitations in GaAs quantum wires. Phys. Rev. Lett., 67, 3298-last pp, ISSN: 00319007

Gupta R., Xiong Q., Mahan G.D. \& Eklund P.C., (2003a). Surface optical phonons in gallium phosphide nanowires. Nano Lett., 3, (Dec 2003) 1745-50, ISSN: 1530-6984

Gupta R., Xiong Q., Adu C.K.,. Kim U.J \& Eklund P.C. (2003b). Laser-induced Fano resonance scattering in silicon nanowires. Nano Lett., 3, (May 2003) 627-31, ISSN: 1530-6984

Harima H. (2002). Properties of GaN and related compounds studied by means of Raman scattering. \& Phys. Condens. Matter,14, (Sep 2002) R967-R993, ISSN: 0953-8984

Hartschuh A., Sanchez E.J., Xie X.S. \& Novotny L. (2003). Near-field second-harmonic generation induced by local field enhancement. Phys. Rev. Lett., 90, (Jan 2003) 095503, ISSN: 0031-9007

Hartschuh A., Pedrosa H.N., Novotny L. \& Krauss T.D. (2003). Simultaneous fluorescence and Raman scattering from single carbon nanotubes. Science, 301, 1354-6, ISSN: 0036-8075

Hu Y., Churchill H.O.H., Reilly D.J., Xiang J., Lieber C.M. \& Marcus C.M. (2007). A Ge/Si heterostructure nanowire-based double quantum dot with integrated charge sensor Nature Nanotech., 2, (Oct 2007) 622-5, ISSN: 1748-3387

Iwasa Y., Arima T., Fleming R.M., Siegrist T., Zhou O., Haddon R.C., Rothberg L.J., Lyons K.B., Carter H.L., Hebard A.F., Tycko R., Dabbagh G., Krajewski J.J., Thomas G.A. \& Yagi T. (1994). New phases of C-60 synthesized at high pressure, Science, 264, (Jun 1994) 1570-2, ISSN: 0036-8075

Jalilian R., Sumanasekera G.U., Chandrasekharan H. \& Sunkara M.K. (2006). Phonon confinement and laser heating effects in Germanium nanowires. Phys. Rev. B 74, (Oct 2006) 155421 ISSN: 1098-0121

Jorio A., Pimenta M., Souza A., Saito R., Dresselhaus G. \& Dresselhaus M. (2003). Characterizing carbon nanotubes samples with resonance Raman scattering. New $d$ Phys., 5, 139, (Oct 2003) 1-17, ISSN: 1367-2630 
Kawashima Y. \& Katagiri G. (1999). Observation of the out-of-plane mode in the Raman scattering from graphite edge plane. Phys. Rev. B, 59, 1, (Jan 1999) 62-4, ISSN: 01631829

Kikkawa J., Ohno Y. \& Takeda S. (2005). Growth rate of Silicon nanowires. Appl. Phys.Lett., 86, 123109, ISSN: 0003-6951

Kim K., Lambrecht W.R.L. \& Segall B. (1996). Elastic constants and related properties of tetrahedrally bonded BN, AlN, GaN, and InN. Phys. Rev. B, 53, (Jun 1996) 16310-26 ISSN: 0163-1829

Kobliska R.J. \& Solin S.A. (1973). Raman Spectrum of Wurtzite Silicon. Phys. Rev. B, 8, (Oct 1973) 3779, ISSN: 0163-1829

Krahne R., Chilla G., Schuller C., Carbone L., Kudera S., Mannarini G., Manna L., Heitmann D. \& Cingolani R. (2006). Confinement effects on optical phonons in polar tetrapod nanocrystals detected by resonant inelastic light scattering. Nano Lett., 6, (Mar 2006) 478-82, ISSN: 1530-6984

Lange H., Mohr M., Artemyev M., Woggon U. \& Thomsen C. (2008). Direct Observation of the Radial Breathing Mode in CdSe Nanorods. Nano Lett., 8, 12, (Nov 2008) 4614-7, ISSN: 1530-6984

Lehmann V. \& Gosele U. (1991). Porous silicon formation - a quantum wire effect. Appl. Phys. Lett., 58, (Feb 1991) 856-8, ISSN: 0003-6951

Li D.Y., Wu Y., Kim P.. Yang P. \& Majumdar A. (2003). Thermal conductivity of Si/SiGe superlattice nanowires. Appl. Phys. Lett., 83, (Oct 2003) 2394-8, ISSN: 0003-6951

Li H.D., Zahng S.L, Yang H.B, Zou G.T., Yang Y.Y., Yue K.T., Wu X.H. \& Yan Y. (2002). Raman spectroscopy of nanocrystalline GaN synthesized by arc plasma. $I$ Appl. Phys., 91, 7, (Apr 2002) 4562-7, ISSN: 0021-8979

Lin H.M., Chen Y.L., Yiang J., Liu Y.C., Yin K.M., Kai J.J., Chen L.C., Chen Y.F. \& Chen C.C. (2003). Synthesis and Characterization of Core-Shell GaP@GaN and GaN@GaP Nanowires. Nano Lett., 3, 4, (Mar 2003) 537-541, ISSN: 1530-6984

Livneh T., Zhang J.P., Cheng G.S. \& Moskovits (2006). Polarized raman scattering from single GaN nanowires. Phys. Rev. B, 74, (Jul 2006) 035320, ISSN: 1098-0121

Long D.A., Raman Spectroscopy. McGraw-Hill, 1977

Lopez F.J., Hemesath E.R. \& Lauhon L.J., (2009) Ordered Stacking Fault Arrays in Silicon Nanowires. Nano Lett., 9, (Jul 2009) 2774-9 ISSN: 1530-6984

Loudon R. (2001). The Raman effect in crystals. Adv. Phys.,50, 7, 813-864, ISSN: 0001-8732

Magidson V. \& Beserman R., (2002). Fano-type interference in the Raman spectrum of photoexcited Si. Phys. Rev. B, 66, (Nov 2002) 195206, ISSN: 1098-0121

Mahan G.D., Gupta R., Xiong Q., Adu C.K. \& Eklund P.C., (2003). Optical phonons in polar semiconductor nanowires. Phys. Rev. B, 68, (Aug 2003) 073402, ISSN: 0163-1829

Maultzsch J., Telg H., Reich S. \& Thomsen C. (2005). Radial breathing mode of single-walled carbon nanotubes: Optical transition energies and chiral-index assignment. Phys. Rev. B, 72, (Nov 2005) 205438, ISSN: 1098-0121

Mishra A., Titova L.V., Hoang T.B., Jackson H.E., Smith L.M., Yarrison-Rice J.M., Kim Y, Joyce H.J., Gao Q., Tan H.H. \& Jagadish C. (2007). Polarization and temperature dependence of photoluminescence from zincblende and wurtzite InP nanowires. Appl. Phys. Lett., (Dec 2007) 91, 263104, ISSN: 0003-6951

Otto A., Mrozek I., Grabhorn H. \& Akemann W., J. (1992). Surface-enhanced Raman scattering. el Phys.: Condens. Matter, 4, (Feb 1992) 1143-1212, ISSN: 0953-8984 
Papadimitriou D. \& Nassiopoulou A.G. (1998). Polarized Raman and photoluminescence study on silicon quantum wires. I Appl. Phys. 84, 2, (Jul 1998) 1059-1063, ISSN: 0021-8979

Park C.H., Cheong B.H., Lee K.H. \& Chang K.J. (1994). Structural and electronic properties of cubic, 2H, 4H, and 6H SiC. Phys. Rev. B, 49, 7, (Feb 1994) 4485-93, ISSN: 0163-1829

Pauzauskie P.J., Talaga D., Seo K., Yang P.D. \& Lagugne-Labarthet F. (2005). Polarized Raman confocal microscopy of single gallium nitride nanowires. JA.C.S., 127, 49, (Dec 2005) 17146-7, ISSN: 0002-7863

Pinczuk A., Abstreiter G., Trommer R. \& Cardona M. (1979). Resonance enhancement of Raman-scattering by electron-gas excitations of n-GaAs. Sol. State Comm., 30, 429-32 ISSN: 0038-1098

Pinczuk A., Abstreiter G., Trommer R. \& Cardona M. (1977). Raman-Scattering by wavevector dependent coupled plasmon LO phonons of N-GaAs. Sol. State Comm., 21, 959-62, ISSN: 0038-1098

Piscanec S., Cantoro M., Ferrari A.C., Zapien J. A., Lifshitz Y., Lee S.T., Hofmann S. \& Robertson J. (2003). Raman spectroscopy of silicon nanowires. Phys. Rev. B, 68, (Dec 2003) 241312, ISSN: 1098-0121

Prades J.D., Cirera A., Arbiol J., Morante J.R. \& Fontcuberta i Morral A. (2007). Concerning the $506 \mathrm{~cm}^{-1}$ band in the Raman spectrum of silicon nanowires. Appl. Phys. Lett., 91, (Sep 2007) 123107 , ISSN: 0003-6951

Raffy C., Furthmüller J. \& Bechstedt F. (2002). Properties of interfaces between cubic and hexagonal polytypes of silicon carbide. I Phys.: Condens. Matter, 14, (Nov 2002) 12725-31, ISSN: 0953-8984

Raman C.V. \& Krishnan K.S. (1928). A new type of secondary radiation. Nature, 121, (Jan 1928) 501-2, ISSN: 0028-0836

Rao A.M., Richter E., Bandow S., Chase B., Eklund P.C., Williams K.A., Fang S., Subbasawamy, M. Menon, A. Thess, R.E. Smalley, G. Dresselhaus \& M.S. Dresselhaus K.R (1997). Diameter-selective Raman scattering from vibrational modes in carbon nanotubes. Science, 275, (Jan 1997) 187-191 ISSN: 0036-8075

Reithmaier J.P., Hoger R., Riechert H., Heberle A., Abstreiter G. \& Weimann G. (1990). Band offset in elastically strained InGaAs GaAs multiple quantum wells determined by optical-absorption and electronic Raman-scattering. Appl. Phys. Lett., 56, (Feb 1990) 536-8, ISSN: 0003-6951

Richter H., Wang Z.P. \& Ley L. (1981). The one phonon Raman-spectrum in microcrystalline silicon. Solid State. Commun., 39, 625-9, ISSN: 0038-1098

Roca E., Tralleroginer C. \& Cardona M. (1994). Polar optical vibrational modes in quantum dots. Phys. Rev. B, 49, (May 1994) 13704 ISSN: 0163-1829

Shorubalko I., Leturcq R., Pfund A., Tyndall D., Krischek R., Schön S. \& Ensslin K. (2008). Self-aligned charge read-out for InAs nanowire quantum dots. Nano letters, 8, (Feb 2008) 382-5, ISSN: 1530-6984

Schuller C., Biese G., Keller K., Steinebach C., Heitmann D., Grambow P. \& Eberl K. (1996). Single-particle excitations and many-particle interactions in quantum wires and dots. Phys. Rev. B, 54, (Dec 1996) 17304-7, ISSN: 1098-0121

Shan C.X., Liu Z., Zhang X.T., Wong C.C. \& Hark S.K.. (2006). Wurtzite ZnSe nanowires: growth, photoluminescence, and single-wire Raman properties. Nanotechnology, 17, (Oct 2006) 5561-4, ISSN: 0957-4484 
Steinbach C., Krahne R., Biese G., Schuller C., Heitmann D. \& Eberl K. (1996). Internal electron-electron interactions in one-dimensional systems detected by Raman spectroscopy. Phys. Rev. B, 54, (Nov 1996) 14281-4, ISSN: 0163-1829

Sood A.K., Menendez J., Cardona M. \& Ploog K. (1985). Resonance Raman-scattering by confined LO and TO phonons in GAAs-AlAs superlattices. Phys. Rev. Lett. ,54, 21114, ISSN: 0163-1829

Somers R.C., Bawendi M.G. \& Nocera D.G. (2007). CdSe nanocrystal based chem-/biosensors. Chem. Soc. Rev., 36, 579-591 ISSN: 0306-0012

Spitzer J., Ruf T., Cardona M., Dondl W., Schorer R., Abstreiter G. \& Haller E.E. (1994). Raman-scattering by optical phonons in isotopic $70 \mathrm{Ge}(\mathrm{n}) 74 \mathrm{Ge}(\mathrm{n})$ superlattices. Phys. Rev. Lett., 72, (Mar 1994) 1565-8, ISSN: 0306-0012

Spirkoska D., Abstreiter G. \& Fontcuberta i Morral A. (2008). Size and environment dependence of surface phonon modes of gallium arsenide nanowires as measured by Raman spectroscopy. Nanotechnology, 19, (Oct 2008) 435704, ISSN: 0957-4484

Spirkoska D., Arbiol j., Gustafsson A., Conesa-Boj S., Zardo I., Heigoldt M., Gass M.H., Bleloch A.L., Estrade S., Kaniber M., Rossler J., Peiro F., Morante J.R., Abstreiter G., Samuelson L. \& Fontcuberta i Morral A. (2009). Structural and optical properties of high quality zinc-blende/wurtzite GaAs heteronanowires. Submitted to Phys. Rev. B, (2009) arXiv/cond-mat 0907.1444

Steckel J. S., Coe-Sullivan S., Bulovic V. \& Bawendi M.G. (2003). $1.3 \mathrm{mu}$ m to $1.55 \mathrm{mu} \mathrm{m}$ tunable electroluminescence from PbSe quantum dots embedded within an organic device. Adv. Mater., 15, (Nov 2003) 18626, ISSN: 0935-9648

Thonhauser T. \& Mahan G.D. (2005). Predicted Raman spectra of Si [111] nanowires. Phys. Rev. B, 71, (Feb 2005) 081307, ISSN: 1098-0121

Thunich S., Prechtel L., Spirkoska D., Abstreiter G., Fontcuberta i Morral A. \& Holleitner A. W. (2009). Photocurrent and photoconductance properties of a GaAs nanowire. Appl. Phys. Lett., 95, (Aug 2009) 083111, ISSN: 0003-6951

Turrel G. \& Corset J. (1996). Raman Microscopy Developments and Applications, Academic Press, Harcourt Brace and Company, Malta, ISBN: 0121896900

Ulrichs E., Biese G., Steinebach C., Schuller C., Heitmann D. \& Eberl K. (1997). Onedimensional plasmons in magnetic fields. Phys. Rev. B, 56, (Nov 1997) 12760-3, ISSN: 1098-0121

Vanwees B.J., Vanhouten H., Beenakker C.W.J., Williamson J.G., Kouwenhoven L.P., Vandermarel D. \& Foxon C.T. (1988). Quantized conductance of point contacts in a two dimensional electron gas. Phys. Rev. Lett., 60, (Aug 1988) 848-50, ISSN: 000319007

Wang J., Gudiksen M. S., Duan X., Cui Y. \& Lieber C. M. (2001). Highly Polarized Photoluminescence and Photodetection from Single Indium Phosphide Nanowires. Science, 293, (Aug 2001) 1455-7, ISSN: 0036-8075

Weinstein B.A. \& Piermarini G.J. (1975). Raman-scattering and phonon dispersion in si and GaP at very high-pressure. Phys. Rev. B, 12, 1172-86 ISSN: 0163-1829

Wickham J.N., Herhold A.B. \& Alivisatos A.P. (2000). Shape change as an indicator of mechanism in the high-pressure structural transformations of CdSe nanocrystals. Phys. Rev. Lett., 84, (Jan 2000) 923-6, ISSN: 0031-9007

Wright A.F. (1997) Elastic properties of zinc-blende and wurtzite AlN, GaN, and InN $J$ Appl. Phys., 82, (Sep 2007) 2833-9, ISSN: 0021-8979 
Xiang Y., Cao L., Conesa-Boj S., Estrade S., Arbiol J., Peiro F., Hei $\beta$ M., Zardo I., Morante J.R., Brongersma M.L., \& Fontcuberta i Morral A. (2009). Single crystalline and core-shell indium-catalyzed germanium nanowires-a systematic thermal CVD growth study. Nanotechnology, 20, (Jun 2009) 245608-11, ISSN: 0957-4484

Xiong Q., Wang J., Reese O., Yoon L. C. \& Eklund P.C.. (2004). Raman scattering from surface phonons in rectangular cross-sectional w-ZnS nanowires. Nano Lett. , 4, 10, (Oct 2004) 1991-6, ISSN: 1530-6984

Xiong Q., Chen G., Gutierrex H.R. \& Eklund P.C., (2006). Raman scattering studies of individual polar semiconducting nanowires: phonon splitting and antenna effects Appl. Phys. A Vol. 85, (Nov 2006), 299-305, ISSN: 0947-8396

Yeh C.Y., Lu Z.W., Froyen S. \& Zunger A. (1992). Zinc-blende - wurtzite polytypism in semiconductors. Phys. Rev. B, 46, 16, (Oct 1992)1086-97, ISSN: 0163-1829

Zardo I., Yu L., Conesa-Boj S., Estradé S., Alet P.J., Rössler J., Frimmer M., Roca i Cabarrocas P., Peiró F., Arbiol J., Morante J.R. \& Fontcuberta i Morral A. (2009a). Gallium assisted plasma enhanced chemical vapor deposition of silicon nanowires. Nanotechnology, 20, (Mar 2009) 155602, ISSN: 0957-4484.

Zardo I., Conesa-Boj S., Peiró F., Morante J.R., Arbiol J., Abstreiter G. \& Fontcuberta i Morral A. (2009b). Raman spectroscopy of wurtzite and zinc-blende GaAs nanowires: polarization dependence, selection rules and strain effects. Submitted to Phys. Rev. B, (2009) arXiv/cond-mat 0910.5266

Zeng H., Cai W., Cao B., Hu J., Li Y. \& Liu P. (2006). Surface optical phonon Raman scattering in $\mathrm{Zn} / \mathrm{ZnO}$ core-shell structured nanoparticles. Appl. Phys. Lett., 88, (May 2006), 181905, ISSN: 0003-6951.

Zunke M., Schorer R., Abstreiter G., Klein W., Weimann G. \& Chamberlain M.P. (1995). Angular-dispersion of confined optical phonons in GaAs/AlAs superlattices studied by micro-Raman spectroscopy. Sol. State. Comm., 93, (Mar 1995) 847-51, ISSN: 0038-1098 


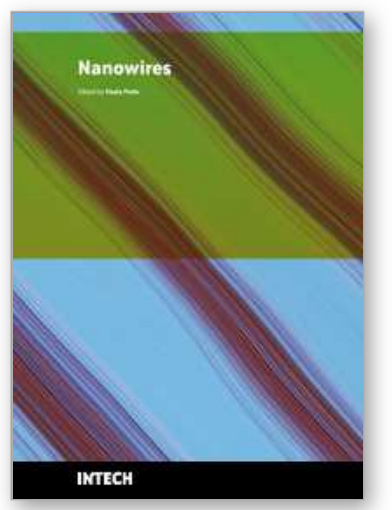

\author{
Nanowires \\ Edited by Paola Prete
}

ISBN 978-953-7619-79-4

Hard cover, 414 pages

Publisher InTech

Published online 01, February, 2010

Published in print edition February, 2010

This volume is intended to orient the reader in the fast developing field of semiconductor nanowires, by providing a series of self-contained monographs focusing on various nanowire-related topics. Each monograph serves as a short review of previous results in the literature and description of methods used in the field, as well as a summary of the authors recent achievements on the subject. Each report provides a brief sketch of the historical background behind, the physical and/or chemical principles underlying a specific nanowire fabrication/characterization technique, or the experimental/theoretical methods used to study a given nanowire property or device. Despite the diverse topics covered, the volume does appear as a unit. The writing is generally clear and precise, and the numerous illustrations provide an easier understanding of the phenomena described. The volume contains 20 Chapters covering altogether many (although not all) semiconductors of technological interest, starting with the IV-IV group compounds ( $\mathrm{SiC}$ and SiGe), carrying on with the binary and ternary compounds of the III-V (GaAs, AIGaAs, GaSb, InAs, GaP, InP, and GaN) and II-VI ( $\mathrm{HgTe}, \mathrm{HgCdTe}$ ) families, the metal oxides ( $\mathrm{CuO}, \mathrm{ZnO}, \mathrm{ZnCoO}$, tungsten oxide, and $\mathrm{PbTiO}$ ), and finishing with $\mathrm{Bi}$ (a semimetal).

\title{
How to reference
}

In order to correctly reference this scholarly work, feel free to copy and paste the following:

Ilaria Zardo, Gerhard Abstreiter and Anna Fontcuberta i Morral (2010). Raman Spectroscopy on Semiconductor Nanowires, Nanowires, Paola Prete (Ed.), ISBN: 978-953-7619-79-4, InTech, Available from: http://www.intechopen.com/books/nanowires/raman-spectroscopy-on-semiconductor-nanowires

\section{INTECH}

open science | open minds

\section{InTech Europe}

University Campus STeP Ri

Slavka Krautzeka 83/A

51000 Rijeka, Croatia

Phone: +385 (51) 770447

Fax: +385 (51) 686166

www.intechopen.com

\section{InTech China}

Unit 405, Office Block, Hotel Equatorial Shanghai

No.65, Yan An Road (West), Shanghai, 200040, China 中国上海市延安西路65号上海国际贵都大饭店办公楼405单元

Phone: +86-21-62489820

Fax: +86-21-62489821 
(C) 2010 The Author(s). Licensee IntechOpen. This chapter is distributed under the terms of the Creative Commons Attribution-NonCommercialShareAlike-3.0 License, which permits use, distribution and reproduction for non-commercial purposes, provided the original is properly cited and derivative works building on this content are distributed under the same license. 